\title{
Transmission of classical and quantum information through a quantum memory channel with damping
}

\author{
Antonio D'Arrigo, ${ }^{1,2}$ Giuliano Benenti, ${ }^{3,4}$ and Giuseppe Falci ${ }^{2,1,5}$ \\ ${ }^{1}$ CNR-IMM-Uos Catania (Università), Consiglio Nazionale delle Ricerche, Via Santa Sofia 64, 95123 Catania, Italy \\ ${ }^{2}$ Dipartimento di Fisica e Astronomia, Universit'a degli Studi Catania, \\ c/o Viale Andrea Doria 6, Ed. 10, 95125 Catania, Italy \\ ${ }^{3}$ CNISM, CNR-INFM \& Center for Nonlinear and Complex Systems, \\ Università degli Studi dell'Insubria, Via Valleggio 11, 22100 Como, Italy \\ ${ }^{4}$ Istituto Nazionale di Fisica Nucleare, Sezione di Milano, via Celoria 16, 20133 Milano, Italy \\ ${ }^{5}$ Centro Siciliano di Fisica Nucleare e di Struttura della Materia (CSFNSM), Viale S. Sofia 64, 95123 Catania, Italy
}

\begin{abstract}
We consider the transfer of classical and quantum information through a memory amplitude damping channel. Such a quantum channel is modeled as a damped harmonic oscillator, the interaction between the information carriers - a train of qubits - and the oscillator being of the Jaynes-Cummings kind. We prove that this memory channel is forgetful, so that quantum coding theorems hold for its capacities. We analyze entropic quantities relative to two uses of this channel. We show that memory effects improve the channel aptitude to transmit both classical and quantum information, and we investigate the mechanism by which memory acts in changing the channel transmission properties.
\end{abstract}

PACS numbers: 03.67.Hk, 03.67.-a, 03.65.Yz

\section{INTRODUCTION}

Noisy channel coding theorems developed in the last fifteen years 1 15] are a central topic in quantum information theory [6, 7]. They provide quantifiers for the effects of noise in quantum communication and allow to investigate how it is possible to reliably communicate through quantum channels. These are mathematically described in the quantum operation $\mathcal{E}$ [6, 7] formalism: an input state described by the density matrix $\rho_{\mathrm{Q}}$ yields an output state $\rho_{Q}^{\prime}=\mathcal{E}\left(\rho_{\mathbf{Q}}\right)$, where the quantum channel $\mathcal{E}$ is a linear map. Effects of noise are described by a non-unitary $\mathcal{E}$.

Such picture can be applied to a variety of situations, for instance to information storage into a quantum memory [8] or to state transfer from one unit to an other of a quantum computer 6, 7]; it may describe photons traveling across a fiber [9] in a quantum cryptographic system or entanglement distribution between different parties $[6,7]$.

A central question is: how is it possible to overcome the information degradation due to noise, in order to obtain a reliable transmission? As in the classical realm, in order to protect communication one may add some redundancy to the information encoding at the channel input [6, 7]. On the other hand redundancy lowers the channel efficiency in terms of information transmitted per channel use. So the former question becomes: "what is the maximum rate of classical or quantum information that can be faithfully transmitted?". Classical and quantum capacities [10], defined as the maximum number of bits/qubits that can be reliably transmitted per channel use (in the asymptotic limit of an infinite number of channel uses), provide the answer to this question.

From the physical side, carriers for quantum or classical information are quantum systems. Noise in communica- tion is due to the unavoidable interaction of such quantum systems with their environment. Since typically a quantum channel has to be used many times, this physical perspective makes clear that the backaction of the environment may play a crucial role in the determination of the maximal rate of faithful information transfer. The simplest models for quantum channels are memoryless, that is to say, at each channel use the environment backaction is negligible and the system undertakes the same transformation $\mathcal{E}_{1}$. If we use the channel $N$ times we have that $\rho_{\mathrm{Q}}^{(N) \prime}=\mathcal{E}_{N}\left(\rho_{\mathrm{Q}}^{(N)}\right)$ where $\mathcal{E}_{N}=\mathcal{E}^{\otimes N}$. On the other hand real systems exhibit correlations among subsequent uses. For example this can happen when environment correlation times are longer than the time between consecutive uses. These kind of channel are called memory channel and for these we have $\mathcal{E}_{N} \neq \mathcal{E}_{1}^{\otimes N}$.

Starting from the seminal work [11], quantum channels with memory have attracted increasing attention in the last years, see [12 25] and references therein. Coding theorems have been proved for classes of quantum memory channels [15, 16]. The presence of memory can bring interesting features: memory can enhance the quantum capacity of a channel [17, 18], and ensemble of entangled states can improve the memory channel aptitude to transmit classical information [11, 25].

Early models of correlated uses considered Markov chains to model memory effects [11, 12, 16]: on one hand this gave a powerful tool to begin to understand how memory effects may change the transmission property of a given channel, on the other hand often real systems exhibit a dynamics which is not captured by simple Markov chains. For instance solid state systems, which are very promising for quantum information purposes thanks to their potential integrability, scalability and controllability, are subject to a variety of noise sources producing low-frequency noise, often exhibiting a typical $1 / f$ spec- 
trum [26]. A realistic description of such systems may require the Hamiltonian modeling of the sources of noise with memory, and in general studying selected Hamiltonian models provides basic insight to understand memory effects of real system used as quantum channels.

In this work we consider a Hamiltonian model implementing a memory amplitude damping channel, and study exactly the behavior for two-channel uses. The amplitude damping channel [6, 7] models for instance environment-induced relaxation processes from the excited state to the ground state of a quantum two-level system. It provides an adequate description of systems where relaxation is dominant with respect to pure dephasing processes.

The Hamiltonian model we choose describes qubit (information carriers) sequentially interacting with a damped harmonic mode. The interaction is of the JaynesCummings type, therefore the whole system can be visualized by a qubit-micromaser [27] system, the qubit train being a stream of two-level Rydberg atoms injected at some rate into the cavity. This model is fundamental in quantum optics, where it describes Cavity [28] Quantum Electrodynamics (QED) systems, where quantum two level systems are strongly coupled to discrete photon modes in high-quality cavities; recently Circuit [29] QED systems operating in the microwave range have been implemented on a solid-state platform and similar architectures have been proposed for the implementation of hybrid quantum memories [8]. A single use yields an an amplitude damping channel 30] $\mathcal{E}_{1}$, provided the cavity is prepared in its ground state. Memory effects naturally arise when repeated uses are considered due the fact that the cavity changes its state after interacting with each information carrier. The strength of memory depends on the cavity quality factor: the greater it is, the stronger are memory effects.

In a previous work we have shown that in the limit of strong dephasing this environment, although decreasing the information transfer per use (the coherent information $I_{C}$, see Sec. II), in a regime where memory effects are present still yields a rate of information transfer larger than operating with a low qubit injection rate to allow memory to decay. In this paper we analyze the channel aptitude to transmit quantum and classical information by considering exactly two uses of this channel. We show that the channel is forgetful [15], and therefore usual quantifiers for quantum communication satisfy noisy coding theorems [1 [5]. A careful study of the above quantities allows to conclude that memory effects improve the channel aptitude to transmit both quantum information, i.e. they may determine a larger $I_{C}$ per use than in the absence of memory. An analogous statement also holds for transmission of classical information.

The paper is organized as follows. In section II we recall the concepts of quantum and classical capacities which apply to memoryless channels and for the class of forgetful memory channels [15]. In section III we present our channel model and demonstrate its forgetfulness. In section IV we discuss the memoryless limit, whereas in section $\mathrm{V}$ we present numerical results for two channel uses, characterizing the channel behavior by suitable entropic quantities; we also investigate the mechanism by which memory changes the channel aptitude to transmit classical and quantum information. Finally in section VI we discuss the significance of our results, pointing out the key role of qubit-environment entanglement in increasing correlations between successive uses.

\section{QUANTUM CAPACITIES}

We consider a quantum information carrier, which is a quantum system $Q$ whose state is chosen from the ensemble $\left\{\sigma_{1}, \ldots, \sigma_{K}\right\}$ ( $\sigma_{i}$ belongs to the Hilbert space $\mathcal{B}_{1}$ of the system), with a priori probabilities $\left\{\xi_{1}, \ldots, \xi_{K}\right\}$. This quantum source is then described by the density operator [31]

$$
\rho_{\mathrm{Q}}=\sum_{i=1}^{K} \xi_{i} \sigma_{i} .
$$

Due to the unavoidable coupling to uncontrollable degrees of freedom, the transmission of this information is in general noisy. Indeed any quantum system interacts with its environment, thus deviating from the expected evolution. Therefore one has to deal with a larger system $\mathrm{QE}$, whose Hamiltonian is $\mathcal{H}=\mathcal{H}_{\mathrm{Q}}+\mathcal{H}_{\mathrm{E}}+\mathcal{H}_{\mathrm{QE}}$, and describes the system $\left(\mathcal{H}_{Q}\right)$, the environment $\left(\mathcal{H}_{\mathrm{E}}\right)$ and their mutual interaction $\left(\mathcal{H}_{Q E}\right)$. The reduced evolution of $Q$ is obtained as a linear, completely positive, trace preserving $(\mathrm{CPT}) \operatorname{map}\left[\right.$ 6, 7] $\mathcal{E}\left(\rho_{\mathrm{Q}}\right)=\operatorname{Tr}_{\mathrm{E}}\left\{\tilde{U}_{\mathrm{QE}}\left(\rho_{\mathrm{Q}} \otimes \omega_{\mathrm{E}}\right) \tilde{U}_{\mathrm{QE}}^{\dagger}\right\}$. Here $\omega_{\mathrm{E}}$ is the initial state of the environment, $\tilde{U}_{\mathrm{QE}}=$ $\exp \left(-i \tilde{\mathcal{H}}_{Q E} t\right)$ and $\tilde{\mathcal{H}}_{Q E}$ is the Hamiltonian of $Q E$ in the interaction picture with respect to $\mathcal{H}_{\mathrm{Q}}$ and $\mathcal{H}_{\mathrm{E}}$. The map $\mathcal{E}$ is the quantum channel, accounting for the noisy processes that the information carrier undergoes during transmission. In what follows we consider $N$ channel uses. The quantum source is still described by (1), where now $\sigma_{i} \in \mathcal{B}_{1}^{\otimes N}$, and the $N$ uses map is denoted by $\mathcal{E}_{N}(\rho)$.

In this paper we are interested to the quantum capacity $Q$ and the classical capacity $C$. The former quantifies the faithful transmission of quantum information, measured by the dimension of the largest subspace of the $N$-use input Hilbert space $\mathcal{B}_{1}^{\otimes N}$ that can be reliably transmitted down the channel, in the limit of large $N$. The classical capacity $C$ gives the maximum amount of classical information that can be reliably transmitted per channel use. That is to say, we wish to identify the largest set of orthogonal input states which remain distinguishable (i.e., orthogonal). In this latter case, the system is not required to preserve the coherence of superpositions of such input states. It is clear that $C \geq Q$, since we can always use orthogonal states in the subspace reliably transmitted to encode classical information. 


\section{A. Memoryless channels}

We first consider memoryless channels, $\mathcal{E}_{N}=\mathcal{E}_{1}^{\otimes N}$, that is each single use map $\mathcal{E}_{1}$ acts independently on the system $\mathrm{Q}$. The quantum capacity $Q$ (measured in qubits per channel use) can be computed as [3, 5, 31]

$$
\begin{gathered}
Q=\lim _{N \rightarrow \infty} \frac{Q_{N}}{N}, \quad Q_{N}=\max _{\rho_{\mathrm{Q}}} I_{c}\left(\mathcal{E}_{N}, \rho_{\mathrm{Q}}\right), \\
I_{c}\left(\mathcal{E}_{N}, \rho_{\mathrm{Q}}\right)=S\left[\mathcal{E}_{N}\left(\rho_{\mathrm{Q}}\right)\right]-S_{e}\left(\mathcal{E}_{N}, \rho_{\mathrm{Q}}\right) .
\end{gathered}
$$

Here $S(\rho)=-\operatorname{Tr}\left[\rho \log _{2} \rho\right]$ is the von Neumann entropy, $S_{e}\left(\mathcal{E}_{N}, \rho_{\mathrm{Q}}\right)$ is the entropy exchange [32], defined as

$$
S_{e}\left(\mathcal{E}_{N}, \rho_{\mathrm{Q}}\right)=S\left[\left(\mathcal{I} \otimes \mathcal{E}_{N}\right)(|\Psi\rangle\langle\Psi|)\right],
$$

where $|\Psi\rangle$ is any purification of $\rho_{\mathrm{Q}}$. That is, we consider the system $Q$, described by the density matrix $\rho_{Q}$, as a part of a larger quantum system $\mathrm{RQ}$ in a pure state $|\Psi\rangle$; the reference system $R$ evolves trivially, according to the identity superoperator $\mathcal{I}$ whereas $\rho_{\mathrm{Q}}=\operatorname{Tr}_{\mathrm{R}}|\Psi\rangle\langle\Psi|$. The quantity $I_{c}\left(\mathcal{E}_{N}, \rho_{\mathrm{Q}}\right)$ is called coherent information [33] and must be maximized over over all input states $\rho$.

The regularization $N \rightarrow \infty$ is necessary since in general $I_{c}$ fails to be subadditive [31] and therefore it cannot be excluded that $Q_{N} / N>Q_{1}$. The regularization is not necessary if the final state of the environment can be reconstructed from the final state of the system. In this case, referred to as degradable channels 34, the coherent information reduces to a suitable conditional entropy, which is subadditive, and the quantum capacity is given by the "single-letter" formula $Q=Q_{1}$. It is worth noticing that for degradable channels the private classical capacity $C_{p}$, defined as the capacity for transmitting classical information protected against an eavesdropper [5], is equal to the quantum capacity $Q$ 35. .

The reliability of transmission of a subspace $\mathcal{B}$ through a channel described by the CPT map $\mathcal{E}$ can be measured by the minimum pure-state fidelity 36.

$$
F_{p}(\mathcal{B}, \mathcal{E}) \equiv \min _{|\psi\rangle \in \mathcal{B}}\langle\psi|\mathcal{E}(|\psi\rangle\langle\psi|)| \psi\rangle
$$

Following [37] we say that the rate (per channel use) $R$ of transmission of subspace dimensions is achievable with channel $\mathcal{E}_{1}$ if there exists a sequence of subspaces $\mathcal{B}^{(N)}$ of $\mathcal{B}_{1}^{\otimes N}$ such that

$$
\limsup _{N \rightarrow \infty} \frac{\log _{2} \operatorname{dim}\left(\mathcal{B}^{(N)}\right)}{N}=R
$$

and there are coding and decoding $\mathrm{CPT}$ maps, $\mathcal{C}^{(N)}$ and $\mathcal{D}^{(N)}$, such that

$$
\lim _{N \rightarrow \infty} F_{p}\left(\mathcal{B}^{(N)}, \mathcal{D}^{(N)} \circ \mathcal{E}_{1}^{\otimes N} \circ \mathcal{C}^{(N)}\right)=1
$$

(for the sake of simplicity, we assume that $\mathcal{E}^{\otimes N}, \mathcal{C}^{(N)}$, and $\mathcal{D}^{(N)}$ all act on the same Hilbert space $\mathcal{B}_{1}^{\otimes N}$ ). The quantum capacity $Q$ of the channel is the supremum of achievable rates $R$. Therefore, $\forall \varepsilon, \delta>0, \exists N_{0} \in \mathbb{N}$ such as, $\forall N>N_{0}, \exists \mathcal{C}^{(N)}, \mathcal{D}^{(N)}, \mathcal{B}^{(N)} \subseteq \mathcal{B}_{1}^{\otimes N}$, with $\left[\log _{2} \operatorname{dim}\left(\mathcal{B}^{(N)}\right)\right] / N \geq Q-\delta$, and we can transmit each state in the subspace $\mathcal{B}^{(N)}$ with high fidelity, i.e., $F_{p}\left(\mathcal{B}^{(N)}, \mathcal{D}^{(N)} \circ \mathcal{E}_{1}^{\otimes N} \circ \mathcal{C}^{(N)}\right) \geq 1-\varepsilon$. That is to say, $\forall|\psi\rangle \in \mathcal{B}^{(N)}$,

$$
\left\langle\psi\left|\mathcal{D}^{(N)} \circ \mathcal{E}_{1}^{\otimes N} \circ \mathcal{C}^{(N)}(|\psi\rangle\langle\psi|)\right| \psi\right\rangle \geq 1-\varepsilon .
$$

The classical capacity is given by [2, 4, 38]

$$
\begin{array}{r}
C=\lim _{N \rightarrow \infty} \frac{C_{N}}{N}, \quad C_{N}=\max _{\left\{\xi_{k}, \sigma_{k}\right\}} \chi\left(\left\{\xi_{k}, \sigma_{k}\right\}\right), \\
\chi\left(\left\{\xi_{k}, \sigma_{k}\right\}\right)=S\left[\mathcal{E}_{N}\left(\rho_{\mathrm{Q}}\right)\right]-\sum_{k} \xi_{k} S\left[\mathcal{E}_{N}\left(\sigma_{k}\right)\right],
\end{array}
$$

with $\rho_{\mathrm{Q}}$ given by (11) and $\chi$ is the Holevo information [6, 7, 39]. Therefore, $C_{N}$ is obtained after maximization of the Holevo information over all possible ensembles $\left\{\xi_{k}, \sigma_{k}\right\}$ which produce $\rho_{\mathrm{Q}}$. Note that for the reliable transmission of classical information the system is not required to preserve relative phases of superpositions of different messages. Therefore, condition (77) must be fulfilled not for all states in subspace $\mathcal{B}^{(N)}$ but just for a orthonormal basis of that subspace. Classical capacity is then obtained as the supremum of achievable rates (per channel use) of transmission of classical information.

\section{B. Memory channels}

When memory effects are taken into account, $\mathcal{E}_{N} \neq$ $\mathcal{E}_{1}^{\otimes N}$, i.e., the channel does not act on each carrier independently. In this case the regularized coherent and Holevo information in general only provide upper bounds on the channel capacities. However, for the class of forgetful channels [15], for which memory effects decay exponentially with time, a quantum coding theorem showing that this bounds can be saturated exists [15].

It is useful to express forgetfulness in an operational way, pointing out the feature that allows the mapping of a forgetful channel into a memoryless one, with negligible error. The key point is the use of a double-blocking strategy. Following Ref. [15] (see also Ref. [17]), we consider blocks of $N+L$ uses of the channel and do the actual coding and decoding for the first $N$ uses, ignoring the remaining $L$ idle uses. The resulting CPT map $\overline{\mathcal{E}}_{N+L}$ actually acts on density matrices $\rho$ on $\mathcal{B}_{1}^{\otimes N}$. Considering $M$ uses of such blocks, the corresponding CPT map $\overline{\mathcal{E}}_{M(N+L)}$ can be approximated, with arbitrarily small error, by the memoryless setting $\overline{\mathcal{E}}_{N+L}^{\otimes M}$ if [15]:

$$
\left\|\overline{\mathcal{E}}_{M(N+L)}\left(\rho_{\mathrm{Q}}\right)-\overline{\mathcal{E}}_{N+L}^{\otimes M}\left(\rho_{\mathrm{Q}}\right)\right\|_{1} \leq h(M-1) c^{-L},
$$

where $\|\rho\|_{1} \equiv \operatorname{Tr} \sqrt{\rho^{\dagger} \rho}$ denotes the trace norm [6], $\rho_{\mathbf{Q}}$ is any $M N$ input state, $h$ and $c$ are real parameters, that 
are independent of the input state $\rho_{\mathrm{Q}}$, with $h>0$ and $c>1$. Expression (10) states that, even though the error committed by replacing the memory channel itself with the corresponding memoryless channel grows with the number of blocks $M$, it goes to zero exponentially fast with the number $L$ of idle uses in a single block. This key feature of forgetful channels allows the proof of coding theorems for this class of quantum memory channels, by mapping them into the corresponding memoryless channels, for which quantum coding theorems hold [15]. In the following, we will use the wording forgetful channels for systems satisfying inequality (10), independently of the specific model used in Ref. [15].

Hereafter we will show, by using arguments similar to the ones used in Ref. [17] for the classical capacity, that the quantum capacity coding theorem holds for forgetful channels, while we refer to [15, 17] for the classical capacity. The coding theorems seen in the previous sections hold for the memoryless channel $\overline{\mathcal{E}}_{N+L}$, and therefore a coding-decoding scheme exists such that $\forall \delta>0$, $\forall \varepsilon>0, \exists M_{0} \in \mathbb{N}$ such that $\forall M>M_{0}$ there exist coding and decoding CPT maps $\overline{\mathcal{C}}^{(M)}$ and $\overline{\mathcal{D}}^{(M)}$ and subspace $\overline{\mathcal{B}}^{(M)} \subseteq \mathcal{B}_{1}^{\otimes M N}$, with $\left[\log _{2} \operatorname{dim}\left(\overline{\mathcal{B}}^{(M)}\right)\right] /(M N) \geq Q-\delta$, and, $\forall|\psi\rangle \in \overline{\mathcal{B}}^{(M)}$,

$$
\left\langle\psi\left|\overline{\mathcal{D}}^{(M)} \circ \overline{\mathcal{E}}_{N+L}^{\otimes M} \circ \overline{\mathcal{C}}^{(M)}(|\psi\rangle\langle\psi|)\right| \psi\right\rangle \geq 1-\varepsilon .
$$

We will prove that the above coding-decoding scheme works well also for a forgetful memory channel. For this purpose, it is useful to observe that (11) is fulfilled provided that

$$
\||\psi\rangle\langle\psi|-\overline{\mathcal{D}}^{(M)} \circ \overline{\mathcal{E}}_{N+L}^{\otimes M} \circ \overline{\mathcal{C}}^{(M)}(|\psi\rangle\langle\psi|) \|_{1} \leq \frac{\varepsilon}{2}
$$

$\forall|\psi\rangle \in \overline{\mathcal{B}}^{(M)}$ [40]. Note that the first member of the inequality (12) is just a measurement of the error probability in transmitting the generic state $|\psi\rangle$. To prove the validity of the coding-decoding scheme for the memory channel, we will show that this error is small also for the channel $\overline{\mathcal{E}}_{M(N+L)}$, namely:

$$
\||\psi\rangle\langle\psi|-\overline{\mathcal{D}}^{(M)} \circ \overline{\mathcal{E}}_{M(N+L)} \circ \overline{\mathcal{C}}^{(M)}(|\psi\rangle\langle\psi|) \|_{1}
$$

can be made arbitrarily small. Due to the triangle inequality, an upper bound to the quantity in (13) is provided by

$$
\begin{aligned}
& \||\psi\rangle\langle\psi|-\overline{\mathcal{D}}^{(M)} \circ \overline{\mathcal{E}}_{N+L}^{\otimes M} \circ \overline{\mathcal{C}}^{(M)}(|\psi\rangle\langle\psi|) \|_{1} \\
& +\| \overline{\mathcal{D}}^{(M)} \circ \overline{\mathcal{E}}_{N+L}^{\otimes M} \circ \overline{\mathcal{C}}^{(M)}(|\psi\rangle\langle\psi|) \\
& \quad-\overline{\mathcal{D}}^{(M)} \circ \overline{\mathcal{E}}_{M(N+L)} \circ \overline{\mathcal{C}}^{(M)}(|\psi\rangle\langle\psi|) \|_{1} .
\end{aligned}
$$

Knowing that (12) holds, we have to prove that the second term in (14) can be made arbitrarily small $\forall|\psi\rangle \in$ $\overline{\mathcal{B}}^{(M)}$. Therefore it is sufficient to prove that

$$
\left\|\overline{\mathcal{D}}^{(M)} \circ \overline{\mathcal{E}}_{N+L}^{\otimes M}\left(\rho_{\mathbf{Q}}\right)-\overline{\mathcal{D}}^{(M)} \circ \overline{\mathcal{E}}_{M(N+L)}\left(\rho_{\mathbf{Q}}\right)\right\|_{1}
$$

can be made small for any input state $\rho_{\mathrm{Q}} \in \mathcal{B}_{1}^{\otimes M N}$. Due to the contractivity of trace-preserving quantum operations [6], this latter quantity is upper bounded by

$$
\left\|\overline{\mathcal{E}}_{N+L}^{\otimes M}\left(\rho_{\mathrm{Q}}\right)-\overline{\mathcal{E}}_{M(N+L)}\left(\rho_{\mathbf{Q}}\right)\right\|_{1}
$$

which in its turn is upper bounded by $h(M-1) c^{-L}$ for a forgetful channel (10). Then, quantity (13) is upper bounded by the sum of the left part of (12) and by $h(M-$ 1) $c^{-L}$ :

$$
\begin{gathered}
\||\psi\rangle\langle\psi|-\overline{\mathcal{D}}^{(M)} \circ \overline{\mathcal{E}}_{M(N+L)} \circ \overline{\mathcal{C}}^{(M)}(|\psi\rangle\langle\psi|) \|_{1} \leq \\
\||\psi\rangle\langle\psi|-\overline{\mathcal{D}}^{(M)} \circ \overline{\mathcal{E}}_{(N+L)}^{\otimes M} \circ \overline{\mathcal{C}}^{(M)}(|\psi\rangle\langle\psi|) \|_{1}+ \\
h(M-1) c^{-L} .
\end{gathered}
$$

As it is stressed in Ref. 17, it is not a priori clear how the right part of inequality (17) can be made arbitrarily small. Indeed to make small the first term in the right part of (17), that is the error of the product code, one needs a large number of block uses $M$, so that (12) holds; but a large $\mathrm{M}$ pushes up the the second term in the right part of (17), since this term is proportional to $M$. Nevertheless, thanks to exponential dependence on the idle uses $L$, both terms can be simultaneously made arbitrarily small, for $M$ large enough. For example, if $L=\varepsilon M$ one simply find that there exists a given $M_{0}^{\prime}$ such that $\forall M>M_{0}^{\prime}$ quantity (13) is less than $\varepsilon$ [41].

It is worth noticing that we demonstrate that the memory channel $\overline{\mathcal{E}}_{N+L}$, can faithful transmit any amount of quantum information supported by the corresponding memoryless channel, whose quantum capacity is given by applying eq. (2) to the map $\mathcal{E}_{N}$. But one can think that the maximum faithful rate achievable by $\mathcal{E}_{N+L}$ is greater than the one of $\overline{\mathcal{E}}_{N+L}$, because with a doubleblock strategy one throws away the information related to the idle uses. But, it is simple to demonstrate that (see Appendix A):

$$
\begin{aligned}
& I_{c}\left(\rho^{(N+L)}, \mathcal{E}_{N+L}\right) \leq \\
& \quad I_{c}\left(\rho^{(N+L)}, \overline{\mathcal{E}}_{N+L}\right)+L \log _{2} \operatorname{dim}\left(\mathcal{H}_{1}\right) .
\end{aligned}
$$

Therefore, the quantum capacity (Eq. (2)) of the two channels $\mathcal{E}_{N+L}$ and $\overline{\mathcal{E}}_{N+L}$ coincides when $\lim _{N \rightarrow \infty}(L / N)=0$. To fulfill this condition, taking into account that we have set $L=\varepsilon M$, we can choose $N=M^{\alpha}$, with $\alpha>1$.

\section{THE MODEL}

We consider a stream of $N$ qubits (the system, Q) interacting with a structured environment, composed by a harmonic oscillator (the local "unconventional" environment [42], O), which in turn is damped due to the coupling with a reservoir. The overall Hamiltonian reads

$$
\begin{aligned}
& \mathcal{H}(t)=\mathcal{H}_{0}+\mathcal{H}_{\mathrm{QO}}+\delta \mathcal{H} \\
& \mathcal{H}_{0}=\mathcal{H}_{\mathrm{Q}}+\mathcal{H}_{\mathrm{O}}=-\frac{\omega}{2} \sum_{k=1}^{N} \sigma_{z}^{(k)}+\nu\left(a^{\dagger} a+\frac{1}{2}\right) \\
& \mathcal{H}_{\mathrm{QO}}=\lambda \sum_{k=1}^{N} f_{k}(t)\left(a^{\dagger} \sigma_{-}^{(k)}+a \sigma_{+}^{(k)}\right) .
\end{aligned}
$$


Here $\sigma_{z}=|g\rangle\langle g|-| e\rangle\langle e|$, being $|g\rangle$ and $|e\rangle$ the two qubit eigenstates, and $\sigma_{+}=|e\rangle\left\langle g\left|, \sigma_{-}=\right| g\right\rangle\langle e|$ are the qubit rising and lowering operators; $a^{\dagger}$ and $a$ are the creation and annihilation operators for the harmonic oscillator. The qubits-oscillator interaction $\mathcal{H}_{\mathrm{QO}}$ is of the JaynesCummings kind, and we take $\lambda$ real and positive and set $\hbar=1$. The coupling is switchable: $f_{k}(t)=1$ when qubit $k$ is inside the channel (transit time $\tau_{p}$ ), $f_{k}(t)=0$ otherwise. The term $\delta \mathcal{H}$ describes both the reservoir's Hamiltonian and the local environment-reservoir interaction and causes damping of the oscillator, within a dissipation time scale $\tau_{d}$. Two consecutive qubits entering the channel are separated by the time interval $\tau$.

We focus on the resonant regime $\nu \sim \omega$, arguably the most significant when describing the coupling to modes inducing damping. We work in the interaction picture, where the effective Hamiltonian is given by $\tilde{\mathcal{H}}=e^{i \mathcal{H}_{0} t}\left(\mathcal{H}_{\mathrm{QO}}+\delta \mathcal{H}\right) e^{-i \mathcal{H}_{0} t}$ (we will omit the tilde from now on).

We study the regime in which the transit time $\tau_{p}$ is smaller than the separation time $\tau$ between two consecutive qubits entering the channel. Such low-rate regime allows us to neglect collective effects such as superradiance, which are expected to be detrimental for the transmission of classical or quantum information.

We assume that oscillator damping is described by the standard master equation (obtained after tracing over the reservoir)

$$
\dot{\rho}_{\mathrm{O}}=\Gamma\left(a \rho_{\mathrm{O}} a^{\dagger}-\frac{1}{2} a^{\dagger} a \rho_{\mathrm{O}}-\frac{1}{2} \rho_{\mathrm{O}} a^{\dagger} a\right) .
$$

The asymptotic decay (channel reset) to the ground state $|0\rangle$ takes place with rate $\Gamma$, so that $\tau_{d}=1 / \Gamma$. We introduce the memory parameter $\mu \equiv \tau_{d} /\left(\tau+\tau_{d}\right)$. By definition $0<\mu<1$ : fast decay $\tau_{d} \ll \tau$ yields the memoryless limit $\mu \ll 1$, whereas $\mu \lesssim 1$ when memory effects come into play.

We studied model (19)-(20) in Ref. 22], in the case in which an additional mechanism of strong dephasing (without relaxation) not included in (20) is added. It was shown that, while the coherent information per channel use is a decreasing function of the degree of memory, the quantum transmission rate, defined as the number of qubits that can be reliably transmitted per unit of time, is enhanced by memory effects. Hereafter we focus on the original model (19)-(20), which is computationally much more demanding but also more interesting, since the interaction with the local environment can entangle initially separable input qubits.

\section{A. Forgetfulness}

As discussed in Sec. (IIB), to prove that quantum and classical capacities are given by Eqs. (2) and (8) it is sufficient to prove that (10) holds. Due to the triangle inequality of trace norm, it is sufficient to show the validity of inequality (10) for $M=2$ (then it follows that this inequality holds for any $M>2$, see Appendix $\mathrm{B}_{\text {): }}$ :

$$
\left\|\overline{\mathcal{E}}_{2(N+L)}\left(\rho_{\mathrm{Q}}\right)-\overline{\mathcal{E}}_{N+L}^{\otimes 2}\left(\rho_{\mathbf{Q}}\right)\right\|_{1} \leq h c^{-L} .
$$

Thanks to contractivity of trace-preserving quantum operations [6], we can bound from above the left-hand side of (21) with

$$
\left\|\overline{\mathcal{F}}_{2(N+L)}\left(\rho_{\mathrm{Q}} \otimes \rho_{\mathrm{O}}\right)-\overline{\mathcal{F}}_{N+L}^{\otimes 2}\left(\rho_{\mathrm{Q}} \otimes \rho_{\mathrm{O}}\right)\right\|_{1},
$$

where $\rho_{O}=|0\rangle\langle 0|$ is the initial (ground) state of the oscillator and quantum operations $\overline{\mathcal{F}}_{j(N+L)}$ are such that, for any integer $j$,

$$
\overline{\mathcal{E}}_{j(N+L)}\left(\rho_{\mathrm{Q}}\right)=\operatorname{Tr}_{\mathrm{O}}\left[\overline{\mathcal{F}}_{j(N+L)}\left(\rho_{\mathrm{Q}} \otimes \rho_{\mathrm{O}}\right)\right] .
$$

Here $\operatorname{Tr}_{O}$ denotes partial trace over the oscillator (we remind the reader that the partial trace is a tracepreserving quantum operation). Note that $\overline{\mathcal{F}}$ is nonunitary since the oscillator is damped. The quantity in Eq. (22) can also be written as

$$
\left\|\overline{\mathcal{F}}_{N+L}\left(\rho_{\mathrm{QO}}^{\prime}\right)-\overline{\mathcal{F}}_{N+L}\left[\pi_{0}\left(\rho_{\mathrm{QO}}^{\prime}\right)\right]\right\|_{1},
$$

where $\rho_{Q O}^{\prime}$ denotes the, generally entangled, state of system ( $2 N$ qubits) and oscillator, after the first $N+L$ channel uses ( $L$ idle uses follow the crossing of the channel by the first $N$ qubits). The operator

$$
\pi_{0}\left(\rho_{\mathrm{QO}}^{\prime}\right)=\operatorname{Tr}_{\mathrm{O}}\left[\rho_{\mathrm{QO}}^{\prime}\right] \otimes|0\rangle\langle 0|
$$

removes any correlation established between the qubits and the oscillator, and resets the oscillator to its ground state (memoryless setting). In the following we call $\rho_{\mathrm{Q}}^{\prime}=\operatorname{Tr}_{\mathrm{O}}\left[\rho_{\mathrm{QO}}^{\prime}\right]$ the state of the $2 N$-qubit system after the ressetting operation $\pi_{0}$.

We take again advantage of the fact that trace preserving operations are contractive to upper bound the quantity in (24) with

$$
\left\|\rho_{\mathrm{QO}}^{\prime}-\pi_{0}\left(\rho_{\mathrm{QO}}^{\prime}\right)\right\|_{1}=\| \rho_{\mathrm{QO}}^{\prime}-\rho_{\mathrm{Q}}^{\prime} \otimes|0\rangle\langle 0| \|_{1} .
$$

We also define the state:

$$
\tilde{\rho}_{Q}^{\prime}=\frac{1}{w_{0}^{\prime}}\left\langle 0\left|\rho_{Q O}^{\prime}\right| 0\right\rangle,
$$

where $w_{n}^{\prime}=\operatorname{Tr}_{\mathrm{QO}}\left[\Pi_{n} \rho_{\mathrm{QO}}^{\prime} \Pi_{n}\right]$ and $\Pi_{n} \equiv|n\rangle\langle n|: w_{n}^{\prime}$ denotes the population, for the state $\rho_{\mathrm{QO}}^{\prime}$, of the $n$-th energy eigenstate of the harmonic oscillator. By using the triangle inequality we can write:

$$
\begin{aligned}
& \| \rho_{\mathrm{QO}}^{\prime}-\rho_{\mathrm{Q}}^{\prime} \otimes|0\rangle\langle 0| \|_{1} \leq \\
& \quad \| \rho_{\mathrm{QO}}^{\prime}-\tilde{\rho}_{\mathrm{Q}}^{\prime} \otimes|0\rangle\langle 0|\left\|_{1}+\right\| \tilde{\rho}_{\mathrm{Q}}^{\prime}-\rho_{\mathrm{Q}}^{\prime} \|_{1} .
\end{aligned}
$$

We start by considering the first term in the right member of (28). Using Uhlmann's fidelity, $F\left(\rho, \rho^{\prime}\right)=$ $\operatorname{Tr} \sqrt{\rho^{1 / 2} \rho^{\prime} \rho^{1 / 2}}$, and the inequality $\left\|\rho-\rho^{\prime}\right\|_{1} \leq$ $2 \sqrt{1-\left[F\left(\rho, \rho^{\prime}\right)\right]^{2}}[\underline{6}]$ between this fidelity and the trace norm, we obtain

$$
\| \rho_{\mathrm{QO}}^{\prime}-\tilde{\rho}_{\mathrm{Q}}^{\prime} \otimes|0\rangle\langle 0| \|_{1} \leq 2 \sqrt{1-\left\{F\left[\rho_{\mathrm{QO}}^{\prime}, \tilde{\rho}_{\mathrm{Q}}^{\prime} \otimes|0\rangle\langle 0|\right]\right\}^{2}} .
$$


Now we observe that:

$$
\begin{aligned}
& \left(\tilde{\rho}_{\mathrm{Q}}^{\prime} \otimes|0\rangle\langle 0|\right)^{1 / 2} \cdot \rho_{\mathrm{QO}}^{\prime} \cdot\left(\tilde{\rho}_{\mathrm{Q}}^{\prime} \otimes|0\rangle\langle 0|\right)^{1 / 2}= \\
& \quad\left(\tilde{\rho}_{\mathrm{Q}}^{\prime}\right)^{1 / 2} \cdot\left(I_{\mathrm{Q}} \otimes|0\rangle\langle 0|\right) \rho_{\mathrm{QO}}^{\prime}\left(I_{\mathrm{Q}} \otimes|0\rangle\langle 0|\right) \cdot\left(\tilde{\rho}_{\mathrm{Q}}^{\prime}\right)^{1 / 2}= \\
& \quad\left(\tilde{\rho}_{\mathrm{Q}}^{\prime}\right)^{1 / 2} \cdot w_{0}^{\prime} \tilde{\rho}_{\mathrm{Q}}^{\prime} \otimes|0\rangle\langle 0| \cdot\left(\tilde{\rho}_{\mathrm{Q}}^{\prime}\right)^{1 / 2}= \\
& \quad w_{0}^{\prime}\left(\tilde{\rho}_{\mathrm{Q}}^{\prime}\right)^{2} \otimes|0\rangle\langle 0| .
\end{aligned}
$$

By substituting this equality into Eq. (29), we have

$$
\| \rho_{Q O}^{\prime}-\tilde{\rho}_{Q}^{\prime} \otimes|0\rangle\langle 0| \|_{1} \leq 2 \sqrt{1-w_{0}^{\prime}} .
$$

We now consider the second term of the right member of Eq. (28). We observe that we can write $\rho_{Q}^{\prime}$ as

$$
\rho_{\mathrm{Q}}^{\prime}=\sum_{n=0}^{\infty}\left\langle n\left|\rho_{\mathrm{Q}}^{\prime}\right| n\right\rangle=\sum_{n} w_{n}^{\prime} \rho_{\mathbf{Q}_{n}}^{\prime}
$$

where $\rho_{Q_{n}}^{\prime}=\frac{1}{w_{n}^{\prime}}\left\langle n\left|\rho_{Q}^{\prime}\right| n\right\rangle ;$ consequently, $\tilde{\rho}_{\mathrm{Q}}^{\prime}=\rho_{\mathrm{Q}_{0}}^{\prime}$. Therefore [ $[\underline{]}$

$$
\begin{aligned}
& \left\|\tilde{\rho}_{\mathrm{Q}}^{\prime}-\rho_{\mathrm{Q}_{\mathrm{Q}}}^{\prime}\right\|_{1} \leq \sum_{n=0}^{\infty} w_{n}^{\prime}\left\|\rho_{\mathrm{Q}_{n}}^{\prime}-\rho_{\mathrm{Q}_{0}}^{\prime}\right\|_{1}= \\
& \sum_{n \geq 1}^{\infty} w_{n}^{\prime}\left\|\rho_{\mathrm{Q}_{n}}^{\prime}-\rho_{\mathrm{Q}_{0}}^{\prime}\right\|_{1} \leq 2 \sum_{n \geq 1}^{\infty} w_{n}^{\prime}=2\left(1-w_{0}^{\prime}\right) \leq \\
& \quad 2 \sqrt{1-w_{0}^{\prime}}
\end{aligned}
$$

Adding inequalities (31) and (33), we obtain

$$
\| \rho_{\mathrm{QO}}^{\prime}-\rho_{\mathrm{Q}}^{\prime} \otimes|0\rangle\langle 0| \|_{1} \leq 4 \sqrt{1-w_{0}^{\prime}} .
$$

Moreover we observe that

$$
\begin{aligned}
& \sqrt{1-w_{0}^{\prime}}=\sqrt{\sum_{n=1}^{\infty} w_{n}^{\prime}} \leq \sqrt{\sum_{n=0}^{\infty} n w_{n}^{\prime}} \\
& =\sqrt{\left\langle a^{\dagger} a\right\rangle[(N+L) \tau]}=e^{-L \Gamma \tau / 2} \sqrt{\left\langle a^{\dagger} a\right\rangle(N \tau)} .
\end{aligned}
$$

Since $\left\langle a^{\dagger} a\right\rangle$ can at most grow by one as a result of the unitary interaction with a qubit while it drops exponentially at rate $\Gamma$ due to dissipation, we have

$$
\left\langle a^{\dagger} a\right\rangle[(k+1) \tau] \leq\left\langle a^{\dagger} a\right\rangle(k \tau) e^{-\Gamma \tau}+1 .
$$

Therefore, $\left\langle a^{\dagger} a\right\rangle(k \tau)<B$ for any $k$, with $B=1 /(1-$ $\left.e^{-\Gamma \tau}\right)$. This inequality can be inserted into Eq. (34)-(35), thus obtaining

$$
\| \rho_{Q \mathrm{O}}^{\prime}-\rho_{\mathrm{Q}}^{\prime} \otimes|0\rangle\langle 0| \|_{1}<4 \sqrt{B} e^{-L \Gamma \tau / 2},
$$

which implies that inequality (21) is fulfilled and therefore quantum and classical capacities can be computed from the maximization (2) and (8) of coherent and Holevo information, respectively.

Note that our proof is valid independently of the relation between the relevant time scales involved in model (19), namely $\tau_{p}, \tau$, and $\tau_{d}$.

\section{THE MEMORYLESS LIMIT}

It is instructive to consider the memoryless limit $\tau_{d} \ll$ $\tau(\mu \ll 1)$ : damping acts as a built-in reset for the oscillator to its ground state $\rho_{\mathrm{O}}(0)=|0\rangle\langle 0|$ after each channel use. In what follows, we show that for $\tau_{d} \ll \tau$ our model reduces to a memoryless amplitude-damping channel.

\section{A. Without damping}

If we further assume that $\tau_{p} \ll \tau_{d}$, we can neglect nonunitary effects in the evolution of the system and the oscillator during the transit time $\tau_{p}$.

We consider a generic single-qubit input state,

$$
\rho_{\mathrm{Q}_{1}}(0)=(1-p)|g\rangle\langle g|+r| g\rangle\left\langle e\left|+r^{\star}\right| e\right\rangle\langle g|+p| e\rangle\langle e|,
$$

with $p$ real and $|r| \leq \sqrt{p(1-p)}$. Given the initial, separable qubit-oscillator state $\rho_{\mathrm{Q}_{1}}(0) \otimes \rho_{\mathrm{O}}(0)$, we have

$$
\mathcal{E}_{1}\left[\rho_{\mathrm{Q}_{1}}(0)\right]=\operatorname{Tr}_{\mathrm{O}}\left\{U\left(\tau_{p}\right)\left[\rho_{\mathrm{Q}_{1}}(0) \otimes \rho_{\mathrm{O}}(0)\right] U^{\dagger}\left(\tau_{p}\right)\right\},
$$

with $U\left(\tau_{p}\right)$ unitary time-evolution operator determined by the undamped Jaynes-Cummings Hamiltonian:

$$
\left\{\begin{array}{l}
U\left(\tau_{p}\right)|g, 0\rangle=|g, 0\rangle \\
U\left(\tau_{p}\right)|e, 0\rangle=\cos \left(\lambda \tau_{p}\right)|e, 0\rangle-i \sin \left(\lambda \tau_{p}\right)|g, 1\rangle,
\end{array}\right.
$$

with $\{|0\rangle,|1\rangle, \ldots\}$ eigenstates of the harmonic oscillator and $\lambda$ frequency of the Rabi oscillations between levels $|e, 0\rangle$ and $|g, 1\rangle$. It is then easy to obtain [30], in the $\{|g\rangle,|e\rangle\}$ basis,

$$
\mathcal{E}_{1}\left[\rho_{Q_{1}}(0)\right]=\left[\begin{array}{cc}
1-p \cos ^{2}\left(\lambda \tau_{p}\right) & r \cos \left(\lambda \tau_{p}\right) \\
r^{\star} \cos \left(\lambda \tau_{p}\right) & p \cos ^{2}\left(\lambda \tau_{p}\right)
\end{array}\right] .
$$

Eq. (41) corresponds to an amplitude-damping channel:

$$
\begin{aligned}
& \mathcal{E}_{1}\left[\rho_{\mathrm{Q}_{1}}(0)\right]=\sum_{k=0}^{1} E_{k} \rho_{\mathrm{Q}_{1}}(0) E_{k}^{\dagger}, \\
& E_{0}=|g\rangle\langle g|+\sqrt{\eta}| e\rangle\left\langle e\left|, \quad E_{1}=\sqrt{1-\eta}\right| g\right\rangle\langle e|,
\end{aligned}
$$

with $E_{0}, E_{1}$ Kraus operators [6, 7] and $\eta=\cos ^{2}\left(\lambda \tau_{p}\right) \in$ $[0,1]$.

This channel is degradable [43] and therefore to compute its quantum capacity it is sufficient to maximize the coherent information over a single use of the channel. Maximization is achieved by diagonal states $(r=0)$ and one obtains [43]

$$
Q= \begin{cases}\max _{p \in[0,1]}\left\{H_{2}(\eta p)-H_{2}[(1-\eta) p]\right\} & \text { if } \eta \geq \frac{1}{2}, \\ 0 & \text { if } \eta \leq \frac{1}{2},\end{cases}
$$

where $H_{2}(x)=-x \log _{2} x-(1-p) \log _{2}(1-x)$ is the binary Shannon entropy. Since the memoryless amplitudedamping channel is degradable, the private classical capacity $C_{p}$ is equal to the quantum capacity $Q$ [35]. 
The classical capacity $C_{1}$, that is, the maximum amount of classical information that can be reliably transmitted using only encodings that are not entangled over successive uses of the channel, is given by [43]

$$
C_{1}=\max _{p \in[0,1]}\left\{H_{2}(\eta p)-H_{2}\left(\frac{1+\sqrt{1-4 \eta(1-\eta) p^{2}}}{2}\right)\right\} \text {. }
$$

The quantity $C_{1}$ is a lower bound for the classical capacity $C$.

\section{B. With damping}

In this section we show that our memoryless model always reduces to an amplitude-damping channel, independently of the relation between the time scales $\tau_{p}$ and $\tau_{d}$.

The non-unitary evolution of qubit and damped oscillator up to the crossing time time $t=\tau_{p}$ is described by the Lindblad master equation [44]

$$
\begin{aligned}
\frac{d \rho_{\mathrm{QO}}}{d t}=-i\left[\mathcal{H}_{1}, \rho_{\mathrm{QO}}\right] & + \\
L & \rho_{\mathrm{QO}} L^{\dagger}-\frac{1}{2} L^{\dagger} L \rho_{\mathrm{QO}}-\frac{1}{2} \rho_{\mathrm{QO}} L^{\dagger} L,
\end{aligned}
$$

where $\mathcal{H}_{1}$ is the interaction picture Hamiltonian (19) for a single channel use,

$$
\mathcal{H}_{1}=\lambda\left(a^{\dagger} \sigma_{-}+a \sigma_{+}\right),
$$

and the Lindblad operator

$$
L=\sqrt{\Gamma}\left(I_{\mathrm{s}} \otimes a\right)
$$

acts trivially on the qubit while damping the oscillator according to Eq. (20).

The master equation (45) can be solved analytically [30], see Appendix C. We end up with the amplitude-damping channel (42), but with a $\Gamma$ dependent parameter

$$
\eta(\Gamma)=e^{-\Gamma \tau_{p} / 2}\left[\frac{\Gamma}{z} \sinh \left(\frac{z \tau_{p}}{4}\right)+\cosh \left(\frac{z \tau_{p}}{4}\right)\right]^{2},
$$

where $z \equiv \sqrt{\Gamma^{2}-16 \lambda^{2}}$. Note that for $\Gamma=0$ we recover the undamped result, $\eta(0)=\cos ^{2}\left(\lambda \tau_{p}\right)$.

For weak damping, we expand Eq. (48) up to first order in $\Gamma / \lambda$, thus obtaining

$$
\eta(\Gamma)=\eta(0)+\frac{\Gamma}{4 \lambda}\left[\sin \left(2 \lambda \tau_{p}\right)-2 \lambda \tau_{p} \cos ^{2}\left(\lambda \tau_{p}\right)\right] .
$$

Depending on the value of $\lambda \tau_{p}$ we can have either $\eta(\Gamma)>$ $\eta(0)$ or $\eta(\Gamma)<\eta(0)$. In particular, in the case of nearly ideal transmission $\lambda \tau_{p} \ll 1(\eta$ close to 1$)$, we have, to first order in $\lambda \tau_{p}$,

$$
\eta(\Gamma)-\eta(0)=\frac{\Gamma}{6 \lambda}\left(\lambda \tau_{p}\right)^{3}>0 .
$$

We can in this case conclude that a small amount of damping improves the channel capacities $Q$ and $C_{1}$ (indeed $Q$ and $C_{1}$ are growing functions of $\eta[43]$ ). This result is to some extent counterintuitive, as damping can enhance the quality of the channel.

It is interesting to examine also the overdamped limit $\Gamma / \lambda \rightarrow \infty$. In this case we obtain from Eq. (48) that $\eta(\Gamma \rightarrow \infty) \rightarrow 1$, so that the channel becomes noiseless (capacities $Q, C \rightarrow 1$ ). This result is a consequence of the quantum Zeno effect [45]: the strong damping dominates the temporal evolution of the system and the oscillator is frozen in its ground state $|0\rangle$, thus hindering the transition $|e, 0\rangle \rightarrow|g, 1\rangle$. Note that $\lambda$ is the frequency of Rabi oscillations between levels $|e, 0\rangle$ and $|g, 1\rangle$ and $\Gamma=1 / \tau_{d}$ is the inverse of the dissipation time scale. In the Zeno regime $\Gamma / \lambda \gg 1$ dissipation dominates and Rabi oscillations are suppressed. Our model provides a nice illustration of enhancement of quantum channel capacities induced by the Zeno effect.

\section{NUMERICAL RESULTS}

In this section we discuss the results for the case of two channel uses. We numerically resolve the system dynamics related to the Hamiltonian (19) and dissipation (20) via a fourth order Runge-Kutta algorithm 46]. We examine both the coherent information and the Holevo information and show that memory effects allow the channel to exceed the corresponding memoryless quantities. We mainly focus on separable two-qubit input states,

$$
\rho_{\mathrm{Q}_{2}}(0)=\rho_{\mathrm{Q}_{1}}(0)^{\otimes 2},
$$

with $\rho_{\mathrm{Q}_{1}}(0)$ given by Eq. (38). Entangled input states will be briefly discussed at the end of this section.

\section{A. The coherent information}

We start our numerical investigations by computing the coherent information $I_{c}$ for the diagonal input state

$$
\rho_{\mathrm{Q}_{2}}(0)=[(1-\bar{p})|g\rangle\langle g|+\bar{p}| e\rangle\langle e|]^{\otimes 2},
$$

where $\bar{p}$ is the value which, in the memoryless limit, maximizes the coherent information over single uses of the channel (see Eq. (43)). Since the amplitude damping channel is degradable [43], the value obtained from such maximization gives the quantum capacity in the memoryless limit. In Fig. 1, we compute the coherent information, normalized over the number $N=2$ of channel uses, as a function of the time interval $\tau$ between the two qubits entering the channel. We consider weak (main plots) and strong (insets) dissipation strength $\Gamma=1 / \tau_{d}$ and two different values of the transit time $\tau_{p}$, leading to higher- (top) or lower-quality (bottom) channels. The memoryless limit is recovered in the limit $\tau \rightarrow \infty$ (memory parameter $\left.\mu=\tau_{d} /\left(\tau+\tau_{d}\right) \rightarrow 0\right)$, and the memory 


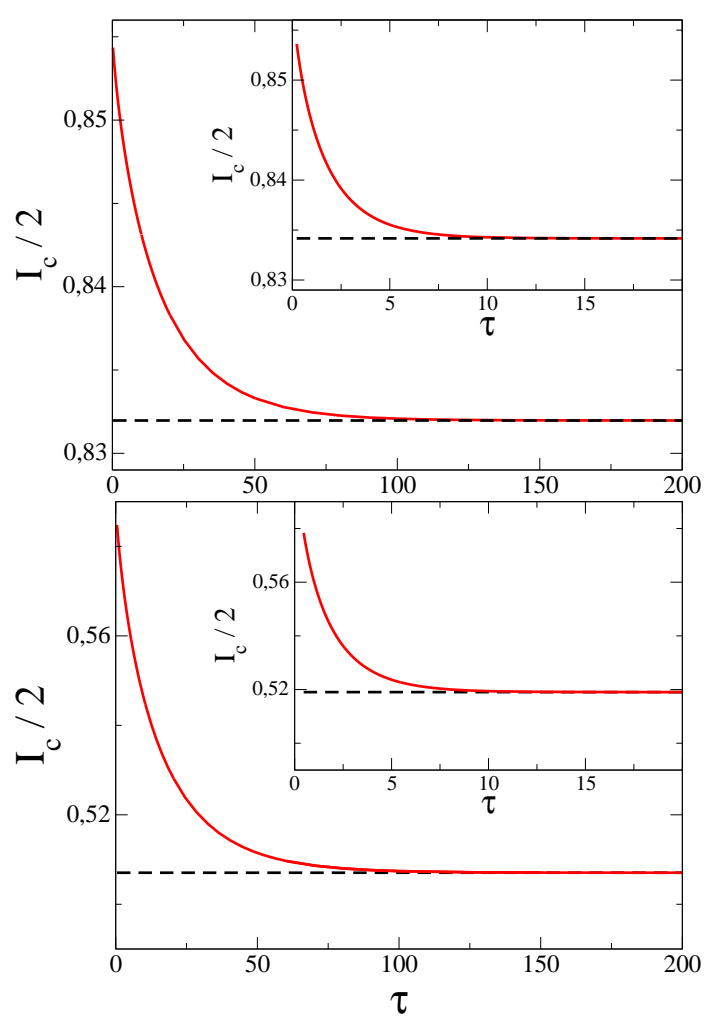

FIG. 1: Coherent information for two channel uses, at coupling strength $\lambda=1, \tau_{p}=0.225$ (above), $\tau_{p}=0.464$ (below), $\Gamma=0.05$ (main plots), $\Gamma=0.5$ (insets), $\eta=0.95$, $\bar{p}=0.4751$, (above, main plot), $\eta=0.95, \bar{p}=0.4754$ (above, inset), $\eta=0.80, \bar{p}=0.4490$ (below, main plot), $\eta=0.81$, $\bar{p}=0.4497$ (below, inset). In each plot the dashed line gives the memoryless quantum capacity $Q$.

parameter is a decreasing function of $\tau$. As discussed in Sec. III] we limit ourselves to the regime $\tau \geq \tau_{p}$, so that the maximum value of the memory parameter is $\tau_{d} /\left(\tau_{d}+\tau_{p}\right)$. In all plots of Fig. 1 it turns out that the coherent information (curves) is a decreasing function of $\tau$, that is, a growing function of the memory parameter $\mu$. We can note that the dependence of the coherent information on $\tau$ (and consequently on the memory parameter $\mu$ ) is of the same kind despite the parameters $\tau_{p}$ and $\Gamma$ take very different values. In the same plots we show the memoryless quantum capacity (dashed lines). From these plots we can conclude that memory effects significantly enhance the coherent information.

The numerical optimization over the separable input states (51) is achieved when $r=0$ (we have checked it for several values of $\tau_{p}$ and $\Gamma$ ) and $p=p_{\mathrm{opt}}$. As an example we show in Fig 2 the dependence of $p_{\text {opt }}$ and $I_{c}\left(p_{\text {opt }}\right)$ on the time separation $\tau$, for dissipation rate $\Gamma=0.5$. As one can see from Fig 2, even though $p_{\text {opt }}$ varies as a function of $\tau$, the improvement of the coherent information with respect to the value of $\bar{p}$ maximizing the memoryless limit is not appreciable.

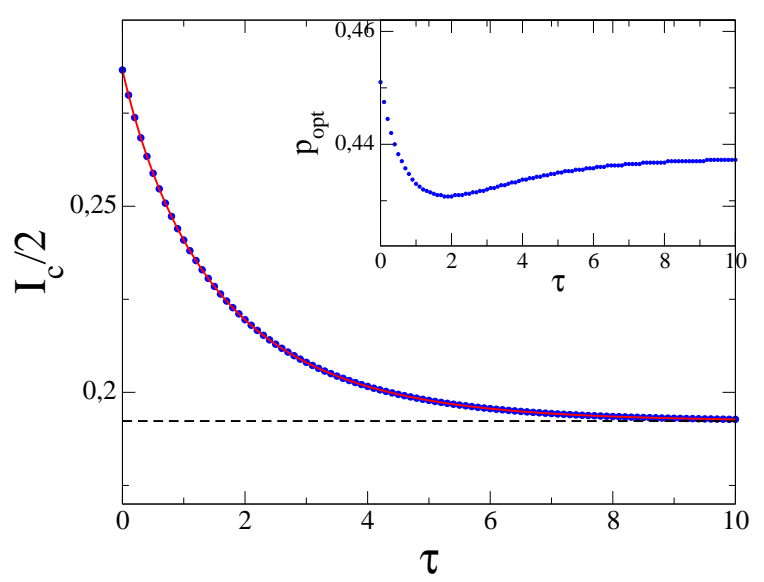

FIG. 2: Coherent information at the optimal input state parameter $p=p_{\text {opt }}$ (main plot) and $p_{\text {opt }}$ (inset) as a function of the time separation $\tau$, for $\lambda=1, \tau_{p}=0.685, \eta=0.62, \Gamma=0.5$ (blue points). The thin red line gives the coherent information for the value of $\bar{p}$ maximizing the memoryless limit. The dashed line corresponds to the memoryless quantum capacity $Q$.

\section{B. The Holevo information}

We numerically compute the Holevo information (9) for separable input states:

$$
\left\{\begin{array}{l}
\sigma_{0}=\left|\psi_{0}\right\rangle\left\langle\psi_{0}|\otimes| \psi_{0}\right\rangle\left\langle\psi_{0}\right|, \\
\sigma_{1}=\left|\psi_{0}\right\rangle\left\langle\psi_{0}|\otimes| \psi_{1}\right\rangle\left\langle\psi_{1}\right|, \\
\sigma_{2}=\left|\psi_{1}\right\rangle\left\langle\psi_{1}|\otimes| \psi_{0}\right\rangle\left\langle\psi_{0}\right|, \\
\sigma_{3}=\left|\psi_{1}\right\rangle\left\langle\psi_{1}|\otimes| \psi_{1}\right\rangle\left\langle\psi_{1}\right|,
\end{array}\right.
$$

with

$$
\left\{\begin{array}{l}
\left|\psi_{0}\right\rangle=\sqrt{1-\tilde{p}}|g\rangle+\sqrt{\tilde{p}}|e\rangle, \\
\left|\psi_{1}\right\rangle=\sqrt{1-\tilde{p}}|g\rangle-\sqrt{\tilde{p}}|e\rangle,
\end{array}\right.
$$

and equal probabilities, $\xi_{k}=\frac{1}{4}(k=0, \ldots, 3)$. In the memoryless limit the ensemble $\left\{\xi_{k}, \sigma_{k}\right\}$ provides the optimal encoding strategy for $C_{1}$ [4], assuming that $\tilde{p}$ is chosen according to Eq. (44).

In Fig. 3 we show that the behavior of the Holevo information $\chi$ is analogous to what obtained above for the coherent information: $\chi$ is a decreasing function of $\tau$, that is, a growing function function of the degree of memory. We have then performed the optimization over the input state parameter $p$. The obtained $p_{\text {opt }}$ and $\chi\left(p_{\text {opt }}\right)$ are shown in Fig. 4 as a function of $\tau$. As for the coherent information, the Holevo quantity does not vary appreciably by substituting the value of $p$ optimizing the memoryless $C_{1}$ with $p_{\text {opt }}$.

As it cannot generally be excluded that $C_{2}>C_{1}$ (or, in the case with memory, that $Q_{2}>Q_{1}$ ), we should optimize over generic two-qubit encodings (or input states for the quantum capacity). Such optimization appears difficult. In what follows, we limit our investigations to a simple ensemble whose states move, when a single parameter 


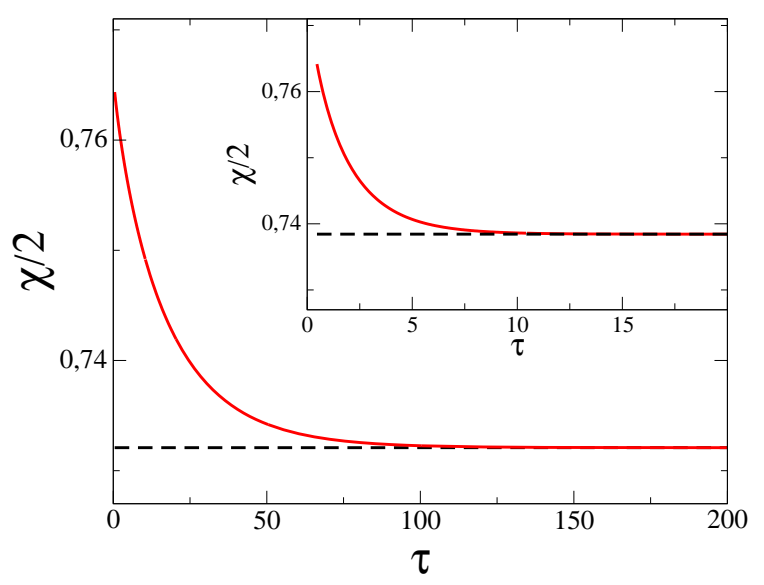

FIG. 3: Holevo information for two channel uses, at $\lambda=1$, $\tau_{p}=0.464, \Gamma=0.05, \eta=0.80, \tilde{p}=0.4329$ (main plot), $\Gamma=0.5, \eta=0.81, \tilde{p}=0.4338$ (inset). In each plot the dashed line gives the memoryless classical capacity $C_{1}$.

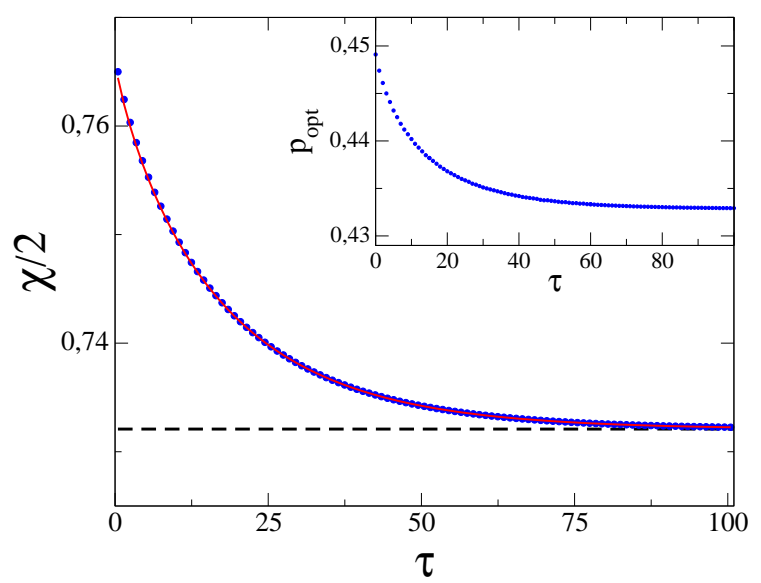

FIG. 4: Holevo information at the optimal input state parameter $p=p_{\text {opt }}$ (main plot) and $p_{\text {opt }}$ (inset) as a function of the time separation $\tau$, for the parameter values of the main plot of Fig. 3. The thin red line is the Holevo information for the value of $\tilde{p}$ maximizing $C_{1}$ in the memoryless limit. The dashed line gives the memoryless classical capacity $C_{1}$.

is varied, from the separable ensemble (53) to an ensemble containing entangled states. We consider equal probabilities, $\xi_{k}=\frac{1}{4}(k=0, \ldots, 3)$ and states $\sigma_{k}=\left|\pi_{k}\right\rangle\left\langle\pi_{k}\right|$, where

$$
\left\{\begin{array}{l}
\left|\pi_{0}\right\rangle=D_{0}\left(\cos \theta\left|\psi_{0}\right\rangle\left|\psi_{1}\right\rangle+\sin \theta\left|\psi_{1}\right\rangle\left|\psi_{0}\right\rangle\right) \\
\left|\pi_{1}\right\rangle=D_{1}\left(\sin \theta\left|\psi_{0}\right\rangle\left|\psi_{1}\right\rangle-\cos \theta\left|\psi_{1}\right\rangle\left|\psi_{0}\right\rangle\right) \\
\left|\pi_{2}\right\rangle=D_{2}\left(\cos \theta\left|\psi_{0}\right\rangle\left|\psi_{0}\right\rangle+\sin \theta\left|\psi_{1}\right\rangle\left|\psi_{1}\right\rangle\right) \\
\left|\pi_{3}\right\rangle=D_{3}\left(\sin \theta\left|\psi_{0}\right\rangle\left|\psi_{0}\right\rangle-\cos \theta\left|\psi_{1}\right\rangle\left|\psi_{1}\right\rangle\right)
\end{array}\right.
$$

with the states $\left|\psi_{0}\right\rangle,\left|\psi_{1}\right\rangle$ given by Eq. (54) and $D_{k}$ normalization constants. The separable ensemble (53) is recovered when $\theta=l \frac{\pi}{2}$, with $l$ integer. Note that in the special case $p=\frac{1}{2}, \theta=\frac{\pi}{4}+m \frac{\pi}{2}$ ( $m$ integer) the states $\left|\pi_{i}\right\rangle$ are maximally entangled Bell states.

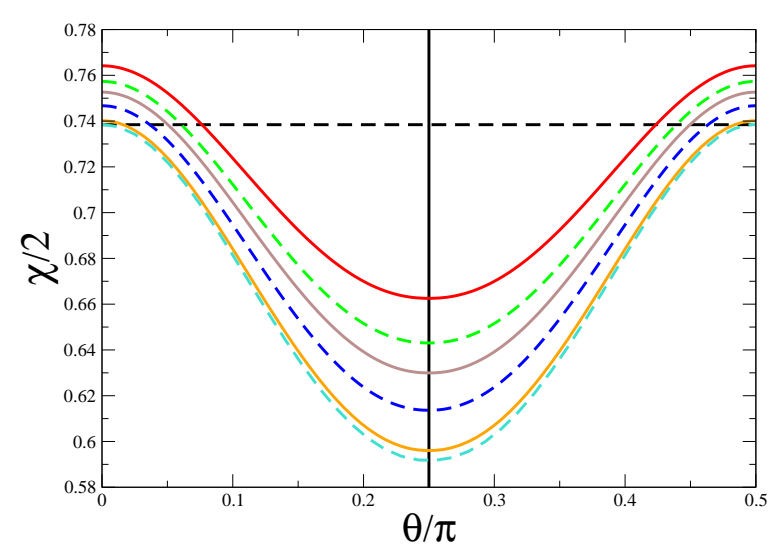

FIG. 5: Holevo information as a function of the parameter $\theta$ determining the ensemble (55), for $\lambda=1, \tau_{p}=0.464$, $\Gamma=0.5, \eta=0.81, \tilde{p}=0.4339$ and, from top to bottom, $\tau-\tau_{p}=0,0.5,1,2,5,10$. The straight dashed line gives the memoryless capacity $C_{1}$.

The Holevo information as a function of $\theta$ is shown in Fig. 5, for several values of the separation time $\tau$. For all considered instances, we always find that $\chi(\theta)$ is optimized by separable input states. Of course we cannot exclude the possibility that different ensembles of entangled states would overcome the capacity $C_{1}$ of separable inputs.

\section{Memory Effects}

We discuss the mechanism which is at the heart of the numerically observed memory-induced enhancement of the coherent information and the Holevo quantity. In our memory channel model, memory not only changes the channel action at each use, but also establishes correlations among the channel outputs. In this section we shall see that the mechanism which permits to overcome the memoryless setting is based on the correlations created between the two systems involved in the communication. In particular, we will demonstrate the key role played by the entanglement between the local environment and the information carriers. Let us first look at quantum information transmission. In the previous section we observed that (see Fig. 1):

$$
\frac{1}{2} I_{c} \geq I_{c, \text { memoryless }}
$$

This relation approaches equality for long times $\tau$ (with respect to $\tau_{d}$ ), i.e. for low values of the memory parameter $\mu$. On the other hand, the difference $\frac{1}{2} I_{c}-I_{c \text {,memoryless }}$ is larger for shorter times $\tau$, i.e. for higher values of $\mu$. Numerically, it turns out that the increasing of $I_{c}$ is 
mainly due to the decreasing of the entropy exchange, since the output entropy depends very slightly on memory.

At first glance, it would be tempting to say that the local environment entangles with the first qubit and therefore its capability to disturb the second qubit is reduced. However, this is not the case. To understand this point we carry out a numerical analysis, in which we consider the entropic quantities related to each single use, and compare them with the corresponding two-uses quantities. We refer to $I_{c, i}\left(S_{e, i}, S_{\text {out }, i}\right)$ as the coherent information (entropy exchange, output entropy) related to $i$-th use $(i=1,2)$, obtained tracing out the degrees of freedom relative to the other channel use. We observe (see Fig. 6) that the coherent information for the second channel use $I_{c, 2}$ is smaller than the memoryless value $I_{c, \text { memoryless: }}$ : memory worsens the performances of the second channel use. For the first use by definition $I_{c, 1}=I_{c \text {,memoryless }}$ (and $S_{e, 1}=S_{e, \text { memoryless). So one has: }}$

$$
\frac{1}{2}\left(I_{c, 1}+I_{c, 2}\right) \leq I_{c, \text { memoryless }}
$$

and because memory only slightly affects $S_{\text {out }}, S_{\text {out }, i}$ (see Fig. 6] above, inset), inequality (57) is mainly due to:

$$
\frac{1}{2}\left(S_{e, 1}+S_{e, 2}\right) \geq S_{e, \text { memoryless }}
$$

That is, if we independently consider the two channel outputs, then the two uses are more noisy than in the memoryless case.

By studying the entropy exchange behavior, we can point out in which way memory improves the channel aptitude to transmit quantum information. From Fig. 6 we have that:

$$
S_{e} \leq S_{e, 1}+S_{e, 2} .
$$

It follows that correlations between systems $R_{1}^{\prime} Q_{1}^{\prime}$ and $\mathrm{R}_{2}^{\prime} \mathrm{Q}_{2}^{\prime}$ satisfy:

$$
\begin{aligned}
& S\left(\mathrm{R}_{1}^{\prime} \mathrm{Q}_{1}^{\prime}: \mathrm{R}_{2}^{\prime} \mathrm{Q}_{2}^{\prime}\right)= \\
& \quad S\left(\mathrm{R}_{1}^{\prime} \mathrm{Q}_{1}^{\prime}\right)+S\left(\mathrm{R}_{2}^{\prime} \mathrm{Q}_{2}^{\prime}\right)-S\left(\mathrm{R}_{1}^{\prime} \mathrm{R}_{2}^{\prime} \mathrm{Q}_{1}^{\prime} \mathrm{Q}_{2}^{\prime}\right)= \\
& \quad S_{e, 1}+S_{e, 2}-S_{e} \geq 0 .
\end{aligned}
$$

(the last equality simply follows from the definitions $S_{e, i}=S\left(\mathrm{R}_{i}^{\prime} \mathrm{Q}_{i}^{\prime}\right)$ and $\left.S_{e}=S\left(\mathrm{R}_{1}^{\prime} \mathrm{R}_{2}^{\prime} \mathrm{Q}_{1}^{\prime} \mathrm{Q}_{2}^{\prime}\right)\right)$. These correlations vanish for the memoryless setting, since by construction systems $R_{1} Q_{1}$ and $R_{2} Q_{2}$ are initially uncorrelated. Therefore memory effects establish correlations between $R_{1}^{\prime} Q_{1}^{\prime}$ and $R_{2}^{\prime} Q_{2}^{\prime}$; moreover such correlations grow with the degree of memory $\mu$. We would like to stress the key role of entanglement between the cavity and the qubits in establishing these correlations. For this purpose, we consider the action of an additional source of noise completely dephasing the local environment before the second channel use [22]. In this case, any entanglement between the cavity and the first qubit is destroyed. The coherent information per channel use
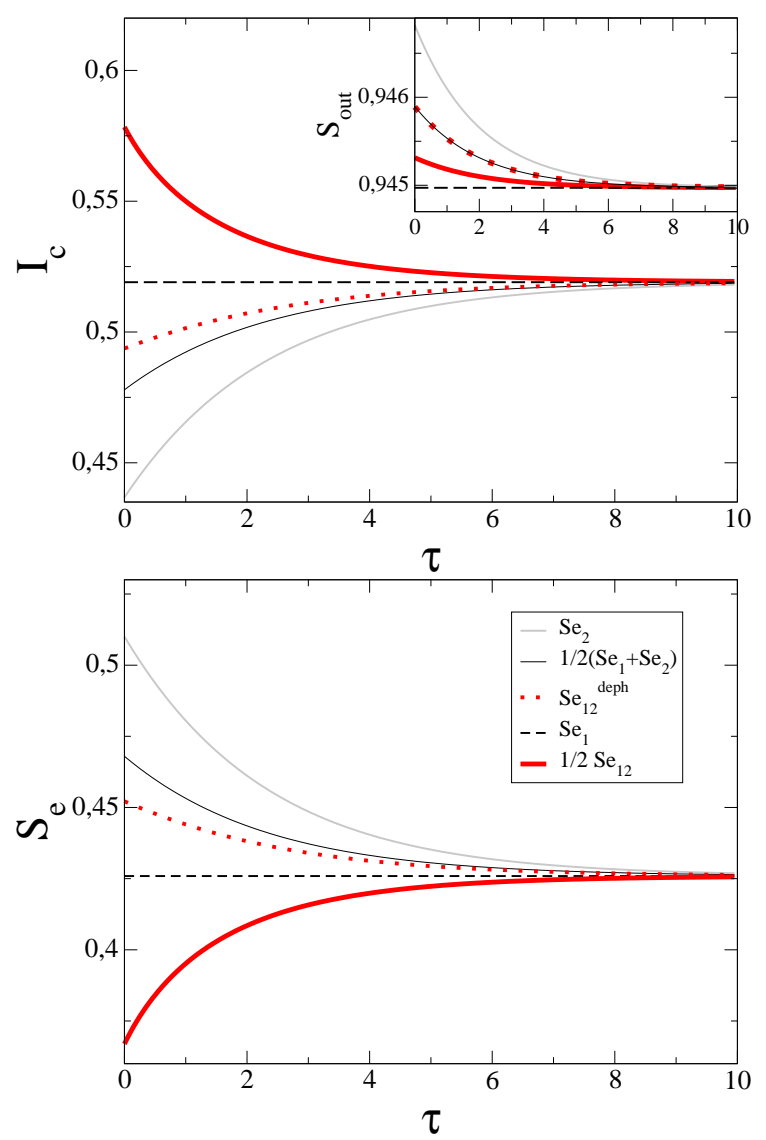

FIG. 6: Above: Coherent information (inset: output entropy) for two channel uses as function of the time $\tau$, at coupling strength $\lambda=1, \tau_{p}=0.464, \Gamma=0.5$; the input state is given by eq. (52) with $p=0.4496$. Thick red curve: $\frac{1}{2} I_{c}$ (in the inset, $\left.\frac{1}{2} S_{\text {out }}\right)$, dashed black line: $I_{c, 1}\left(S_{\text {out }, 1}\right)$, dotted red curve: $\frac{1}{2} I_{c}^{\text {deph }}\left(\frac{1}{2} S_{\text {out }}^{\text {deph }}\right)$, black thin curve: $\frac{1}{2}\left(I_{c, 1}+I_{c, 2}\right)$ $\left(\frac{1}{2}\left(S_{\text {out }, 1}+S_{\text {out }, 2}\right)\right)$, gray curve: $I_{c, 2}\left(S_{\text {out }, 2}\right)$. Note that output entropies range in an interval about fifty times smaller than the one relative to the coherent information. Below: the corresponding entropy exchanges.

$\frac{1}{2} I_{c}^{\text {deph }}$ decreases (see Fig. 6, above) and the inequality (56) is no longer fulfilled: the advantages produced by memory are lost. Returning to the entropy exchange behavior we find that $S_{e}^{\mathrm{deph}}$ approaches $S_{e, 1}+S_{e, 2}$ so that correlations (60) are considerably reduced. We can conclude that the creation of correlations between $R_{1}^{\prime} Q_{1}^{\prime}$ and $\mathrm{R}_{2}^{\prime} \mathrm{Q}_{2}^{\prime}$, mediated by qubit entanglement with the common local environment, is the essential mechanism allowing us to overcome the memoryless quantum capacity.

Let us look at the classical information transmission. We recall the Holevo information expression for a given source $\rho_{\mathrm{Q}}=\sum_{k} \xi_{k} \sigma_{k}$ :

$$
\chi=S\left(\sum_{k} \xi_{k} \sigma_{k}^{\prime}\right)-\sum_{k} \xi_{k} S\left(\sigma_{k}^{\prime}\right)=S_{\text {out }}-\left\langle S_{\text {out }}\right\rangle,
$$

where $\sigma_{k}^{\prime}=\mathcal{E}_{2}\left(\sigma_{k}\right)$ and $\left\langle S_{\text {out }}\right\rangle=\sum_{k} \xi_{k} S\left(\sigma_{k}^{\prime}\right)$ is the output entropy averaged over all codewords. We refer to $\chi_{i}$ 

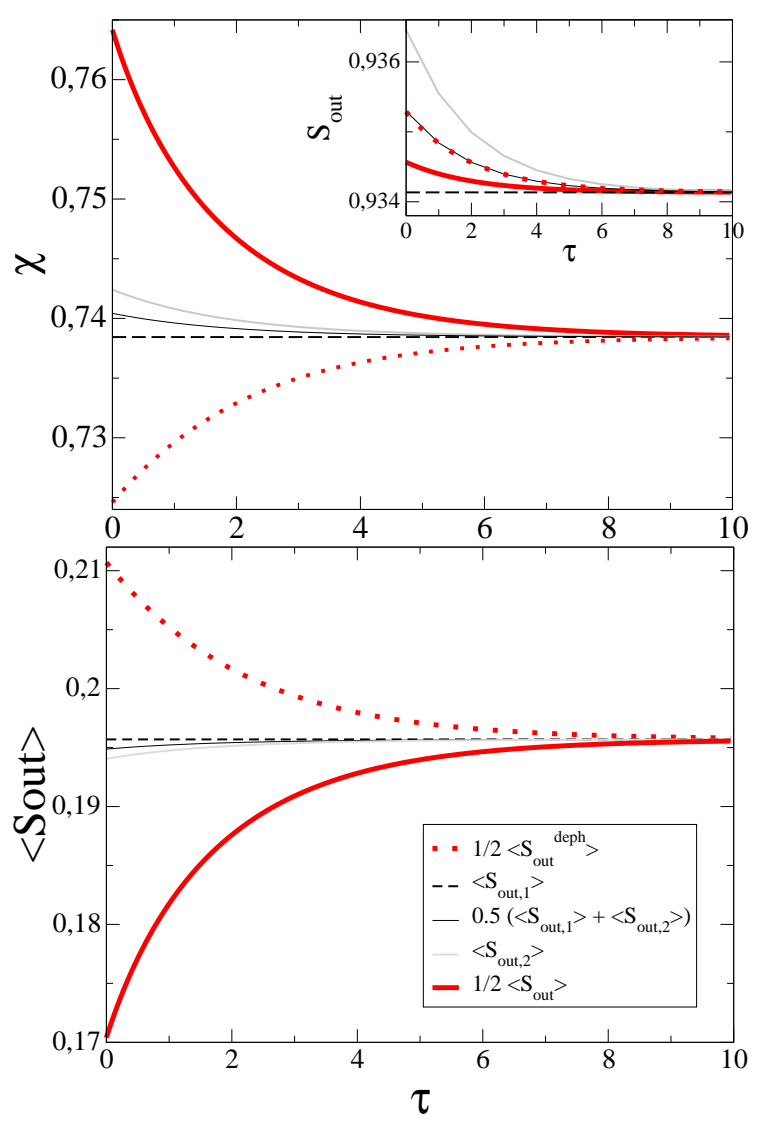

FIG. 7: Above: Holevo quantity (inset: output entropy) for two channel uses as function of the time $\tau$, at coupling strength $\lambda=1, \tau_{p}=0.464, \Gamma=0.5$; the input state is given by eq. (53) with $p=0.4339, \xi_{k}=1 / 4$. Thick red curve: $\frac{1}{2} \chi$ (in the inset, $\left.\frac{1}{2} S_{\text {out }}\right)$, gray curve: $\chi_{2}\left(S_{\text {out }, 2}\right)$, black thin curve: $\frac{1}{2}\left(\chi_{1}+\chi_{2}\right)\left(\frac{1}{2}\left(S_{\text {out }, 1}+S_{\text {out }, 2}\right)\right)$, dashed black line: $\chi_{1}$ $\left(S_{\text {out }, 1}\right)$, dotted red curve: $\frac{1}{2} \chi^{\text {deph }}\left(\frac{1}{2} S_{\text {out }}^{\text {deph }}\right)$. Note that output entropies range in an interval about twenty times smaller than the one relative to the Holevo information. Below: the corresponding average output entropies.

$\left(\left\langle S_{\text {out }, i}\right\rangle, S_{\text {out }, i}\right)$ as the Holevo quantity (average output entropy, output entropy) related to the $i$-th use. Differently from what we have observed for the coherent information, the Holevo quantity related to the second use is not worsened by memory. But the two uses increasing of Holevo information cannot be ascribed to the $\chi_{2}$ behavior. Indeed from Fig. 7 (above), it appears that:

$$
\chi \geq \chi_{1}+\chi_{2} .
$$

As we can see from the same figure (inset) the range of variation of the output entropies $S_{\text {out }}$ and $S_{\text {out }, i}$, is much smaller than the one relative to the Holevo quantity, so that the inequality (62) is mainly due to

$$
\left\langle S_{\text {out }}\right\rangle \leq\left\langle S_{\text {out }, 1}\right\rangle+\left\langle S_{\text {out }, 2}\right\rangle .
$$

We stress that the average output entropy play a role analogous to the entropy exchange in the quantum information transmission scenario: it quantifies the amount of noise introduced by the channel. We can conclude that also from the point of view of classical information transmission, the channel introduces more noise if we independently consider the two channel outputs. Inequality (63) means that, if we send a codeword $\sigma_{i}=\sigma_{i_{1}} \otimes \sigma_{i_{2}}$ down the channel, then the channel establishes correlations between the output states of the two qubits. Also in this case, these correlations are mediated by the local environment: if we completely dephase the cavity after it interacts with the first qubit, we lose any advantage, as one can see from Fig. 7 .

\section{DISCUSSION AND CONCLUSIONS}

We would like to comment on the significance of our results obtained with two channel uses. First of all, one could send a couple of qubits through the channel and reset the oscillator to its ground state after the transit of each couple. In this case our results are exact and show that for this new channel the quantum and classical capacities would be larger than in the memoryless case, where the reset is operated after each channel use. Therefore, our results can be indicative of a trend, that further enhancements of the coherent and Holevo information per channel use may be obtained by considering longer qubit trains.

We can also view the question from a slightly different point of view. We start by considering the correlations between the two-qubit system $Q=Q_{1} Q_{2}$ and the reference system $R=R_{1} R_{2}$ introduced to purify the initial input state (indexes 1 and 2 refer to the first and the second channel use). Such correlations are measured by the quantum mutual information [47]

$$
S(\mathrm{R}: \mathrm{Q})=S(\mathrm{R})+S(\mathrm{Q})-S(\mathrm{RQ}),
$$

where $S$ is the von Neumann entropy and $S(\mathrm{RQ})$ the joint entropy for the composite system RQ. This situation describes the case in which Alice is attempting to transmit two halves (Q) of two pairs of Bell states to Bob, while she retains the other halves (R). After the two channel uses correlations between $\mathrm{R}$ and $\mathrm{Q}$ read as follows:

$$
\begin{aligned}
& S\left(\mathrm{R}^{\prime}: \mathrm{Q}^{\prime}\right)=S\left(\mathrm{R}^{\prime}\right)+S\left(\mathrm{Q}^{\prime}\right)-S\left(\mathrm{R}^{\prime} \mathrm{Q}^{\prime}\right) \\
& \quad=S(\mathrm{R})+S_{\text {out }}-S_{e}=S(\mathrm{R})+I_{c}
\end{aligned}
$$

where the prime symbols refer to the output states, $S_{\text {out }}=S\left(\mathrm{Q}^{\prime}\right)$ is the output entropy and $S_{e}=S\left(\mathrm{R}^{\prime} \mathrm{Q}^{\prime}\right)$ the entropy exchange. Since $S\left(\mathrm{R}^{\prime}\right)=S(\mathrm{R})$ is independent of the degree of memory $\mu$ (i.e., independent of the separation time $\tau$ ), we find that the fact that memory increases coherent information $I_{c}$ is deeply related to the fact that memory helps to preserve correlations between $\mathrm{R}$ and $\mathrm{Q}$.

With regard to the Holevo information, a similar argument can be developed. Indeed, we can model the mixed classical-quantum ensemble $\left\{\xi_{k}, \sigma_{k}\right\}$ by means of an en- 
larged Hilbert space representation [5]:

$$
\rho_{\mathrm{AQ}}=\sum_{k} \xi_{k}|k\rangle\langle k| \otimes \sigma_{k},
$$

where $\{|k\rangle\}$ is and orthonormal basis for the Hilbert space of the auxiliary system A. The correlations between $A$ and $Q$ read

$$
\begin{aligned}
S(\mathrm{~A}: \mathrm{Q})= & S(\mathrm{~A})+S(\mathrm{Q})-S(\mathrm{AQ})= \\
& S(\mathrm{Q})-S(\mathrm{Q} \mid \mathrm{A})= \\
& S\left(\sum_{k} \xi_{k} \sigma_{k}\right)-\sum_{k} \xi_{k} S\left(\sigma_{k}\right)
\end{aligned}
$$

where $S(\mathrm{Q} \mid \mathrm{A})=S(\mathrm{AQ})-S(\mathrm{~A})$ is a conditional von Neumann entropy. Now if we choose an ensemble of pure states $\sigma_{k}$, the correlations between the systems $\mathrm{A}$ and $\mathrm{Q}$ are maximum, and equal to $S(\mathrm{~A})=S\left(\sum_{k} \xi_{k} \sigma_{k}\right)$, since $S\left(\sigma_{k}\right)=0$. But when we transmit the quantum system $Q$ down the channel, it adds noise such that $\sigma_{k} \rightarrow \sigma_{k}^{\prime}=\mathcal{E}_{2}\left(\sigma_{k}\right)$, and in general $S\left(\sigma_{k}^{\prime}\right) \neq 0$. Thus correlations between $\mathrm{A}$ and $\mathrm{Q}$ decrease to:

$$
\begin{aligned}
& S\left(\mathrm{~A}: \mathrm{Q}^{\prime}\right)=S\left(\mathrm{Q}^{\prime}\right)-S\left(\mathrm{Q}^{\prime} \mid \mathrm{A}\right)= \\
& S\left(\sum_{k} \xi_{k} \sigma_{k}^{\prime}\right)-\sum_{k} \xi_{k} S\left(\sigma_{k}^{\prime}\right)=\chi\left(\left\{\xi_{k}, \sigma_{k}\right\}\right)
\end{aligned}
$$

Then the Holevo information is just the correlation between the classical variable we want to transmit and the quantum system we have used to transmit it. Since memory enhances the Holevo quantity with respect to the memoryless setting, we conclude that memory helps to preserve these correlations.

In both scenarios - quantum and classical information transmission - memory helps in preserving the correlations between the systems involved in the transmission. We stress again the key role of entanglement between information carriers and the channel. This effect could be in principle useful for quantum error-correcting codes (QECC). Indeed, understanding in which way memory acts during information transmission, may suggest suitable strategies to design new QECC with performances better than those of standard memoryless QECC. In this perspective, further work will be necessary to see if, in presence of memory, the coherent information and Holevo quantity per channel use increase with the number of channel uses, as one would expect from the data shown in the Figs. 1-3, as it happens for the memory dephasing channel [18].

\section{Acknowledgements}

We acknowledge fruitful discussions with Shash Virmani and an anonymous referee for useful remarks. AD acknowledges L. Bordi, T. D'Arrigo and M. Fici for invaluable help. This work was partially supported by the EU through grant no. PITN-GA-2009-234970, and by the Joint Italian-Japanese Laboratory on "Quantum Technologies: Information, Communication and Computation" of the Italian Ministry of Foreign Affairs.

\section{Appendix A: Quantum capacity of the channel $\overline{\mathcal{E}}_{N+L}$}

We discuss the condition under which the quantum capacity of the channel $\overline{\mathcal{E}}_{N+L}$ is the same as for the $\mathcal{E}_{N+L}$. We consider $n=N+L$ channel use; the related coherent information is:

$$
\begin{aligned}
& I_{c}\left(\mathcal{E}_{n}, \rho^{(n)}\right)= \\
& \quad S\left[\mathcal{E}_{n}\left(\rho^{(n)}\right)\right]-S\left[\left(\mathcal{I}^{(n)} \otimes \mathcal{E}_{n}\right)\left(\left|\Psi^{(n)}\right\rangle\left\langle\Psi^{(n)}\right|\right)\right] .
\end{aligned}
$$

where $\left|\Psi^{(n)}\right\rangle$ is any purification of $\rho^{(n)}$. Now we call $\mathbf{Q}^{(N)}$ and $\mathrm{Q}^{(L)}$ the systems composed by the first $N$ and the last $L$ qubits, respectively. We refer to $\mathrm{R}$ as the purifying system. We observe that:

$$
\mathcal{E}_{n}\left(\rho^{(n)}\right)=\left(\left(\mathcal{I}^{(N)} \otimes \mathcal{N}_{L}\right) \circ\left(\mathcal{E}_{N} \otimes \mathcal{I}^{(L)}\right)\right)\left(\rho^{(N+L)}\right) ;(A
$$

indeed we can image that the overall channel processing on a $n$-input state, can be decomposed in two steps. First, we operate only on the first $N$ use, leaving unaffected the successive $L$ uses $\left(\mathcal{I}_{L}\right.$ is the identity operator): this corresponds to act on the first $N$-uses by the quantum map $\mathcal{E}_{N}$. Second, we perform the quantum operation $\mathcal{N}_{L}$ on the remaining $L$-use, leaving unaffected the first ones, in a such way the overall action on the $n$-input state is still described by $\mathcal{E}_{n}$. By the data processing inequality [31] we have:

$$
I_{c}\left(\mathcal{E}_{n}, \rho^{(n)}\right) \leq I_{c}\left(\mathcal{E}_{N} \otimes \mathcal{I}^{(L)}, \rho^{(n)}\right) .
$$

By definition of coherent information [33],

$$
\begin{aligned}
& I_{c}\left(\mathcal{E}_{N} \otimes \mathcal{I}^{(L)}, \rho^{(n)}\right)= \\
& \quad S\left(\mathcal{E}_{N} \otimes \mathcal{I}^{(L)}\left(\rho^{(n)}\right)\right)- \\
& \quad S\left(\mathcal{I}^{(n)} \otimes\left(\mathcal{E}_{N} \otimes \mathcal{I}^{(L)}\right)\left|\Psi^{(n)}\right\rangle\left\langle\Psi^{(n)}\right|\right),
\end{aligned}
$$

we observe that the last term in the A4 is just the entropy exchange 32 related to the first $N$ channel uses map $\mathcal{E}_{N}$. Indeed $\left|\Psi^{(n)}\right\rangle$, being a purification of $\rho^{(n)}$, it also is a purification of $\rho^{(N)}=\operatorname{Tr}_{Q^{(L)}}\left\{\rho^{(n)}\right\}$, and the map $\mathcal{I}^{(n)} \otimes\left(\mathcal{E}_{N} \otimes \mathcal{I}^{(L)}\right)$ acts on $\mathrm{Q}^{(N)}$ by $\mathcal{E}_{N}$, while does not change the purifying systems $\mathrm{R}$ and $\mathrm{Q}^{(L)}$. Moreover, by the subadditivity of the von Neumann entropy we can bound from above the first term at the second member of (A4):

$$
\begin{aligned}
& S\left(\mathcal{E}_{N} \otimes \mathcal{I}^{(L)}\left(\rho^{(n)}\right)\right) \leq \\
& S\left(\operatorname{Tr}_{Q^{(L)}}\left\{\mathcal{E}_{N} \otimes \mathcal{I}^{(L)}\left(\rho^{(n)}\right)\right\}\right)+ \\
& \quad S\left(\operatorname{Tr}_{Q^{(N)}}\left\{\mathcal{E}_{N} \otimes \mathcal{I}^{(L)}\left(\rho^{(n)}\right)\right\}\right)= \\
& S\left(\mathcal{E}_{N}\left(\rho^{(N)}\right)\right)+S\left(\operatorname{Tr}_{Q^{(N)}}\left\{\mathcal{E}_{N} \otimes \mathcal{I}^{(L)}\left(\rho^{(n)}\right)\right\}\right) \leq \\
& S\left(\mathcal{E}_{N}\left(\rho^{(N)}\right)\right)+L \log _{2} \operatorname{dim}\left(\mathcal{H}_{1}\right),
\end{aligned}
$$


where we used causality [15] in taking the trace with respect to $\mathrm{Q}^{(L)}$ : outputs related to the first $N$ uses do not depend on inputs related to the subsequent $L$ uses. So, returning to the coherent information (A4), we can write:

$$
\begin{aligned}
& I_{c}\left(\mathcal{E}_{N} \otimes \mathcal{I}^{(L)}, \rho^{(n)}\right) \leq \\
& S\left(\mathcal{E}_{N}\left(\rho^{(N)}\right)\right)+L \log _{2} \operatorname{dim}\left(\mathcal{H}_{1}\right)- \\
& \quad S\left(\mathcal{I}^{(n)} \otimes\left(\mathcal{E}_{N} \otimes \mathcal{I}^{(L)}\right)\left|\Psi^{(n)}\right\rangle\left\langle\Psi^{(n)}\right|\right)= \\
& I_{c}\left(\mathcal{E}_{N}, \rho^{(N)}\right)+L \log _{2} \operatorname{dim}\left(\mathcal{H}_{1}\right)
\end{aligned}
$$

Finally by the (A3) and (A6) we have that:

$$
\begin{aligned}
& I_{c}\left(\rho^{(N+L)}, \mathcal{E}_{N+L}\right) \leq \\
& \quad I_{c}\left(\overline{\mathcal{E}}_{N+L}, \rho^{(N+L)}\right)+L \log _{2} \operatorname{dim}\left(\mathcal{H}_{1}\right)
\end{aligned}
$$

since, by the definition of double blocking strategy,

$$
I_{c}\left(\overline{\mathcal{E}}_{N+L}, \rho^{(N+L)}\right)=I_{c}\left(\mathcal{E}_{N}, \rho^{(N)}\right) .
$$

By maximizing over all possible input $\rho^{(n)}=\rho^{(N+L)}$, taking the limit for $N \rightarrow \infty$ and assuming $\lim _{N \rightarrow \infty} L / N=$ 0 , (A7) assures that $Q\left(\mathcal{E}_{N+L}\right) \leq Q\left(\overline{\mathcal{E}}_{N+L}\right)$. On the other hand, the capacity of the channel $\mathcal{E}_{N+L}$ is obviously at least as great as the one of $\overline{\mathcal{E}}_{N+L}$, so one can conclude that $Q\left(\mathcal{E}_{N+L}\right)=Q\left(\overline{\mathcal{E}}_{N+L}\right)$.

\section{Appendix B: Proof of eq. (10) for the channel (19)}

We consider $M$ blocks of $N+L$ uses of the channel (19), in a double blocking strategy perspective (see section (IB). We want to show that:

$$
\left\|\overline{\mathcal{E}}_{M(N+L)}\left(\rho_{\mathrm{Q}}\right)-\overline{\mathcal{E}}_{N+L}^{\otimes M}\left(\rho_{\mathrm{Q}}\right)\right\|_{1} \leq h(M-1) c^{-L} .
$$

We suppose to deal with a larger number $M^{\prime}>M$ of blocks, whose the first $M$ are subjected to the channels operations $\overline{\mathcal{E}}_{M(N+L)}$ or $\overline{\mathcal{E}}_{N+L}^{\otimes M}$, whereas the remaining $M^{\prime}-M$ do not undergo any operation. Note that $M^{\prime}$ is just introduced for technical purpose, in order to carry out the proof. Thanks to the contractivity of tracepreserving quantum operations [6], we can write:

$$
\begin{aligned}
& \left\|\overline{\mathcal{E}}_{M(N+L)}\left(\rho_{\mathrm{Q}}\right)-\overline{\mathcal{E}}_{N+L}^{\otimes M}\left(\rho_{\mathrm{Q}}\right)\right\|_{1} \leq \\
& \| \mathcal{I}_{\left(M^{\prime}-M\right)(N+L)} \otimes \overline{\mathcal{E}}_{M(N+L)}\left(\rho_{\mathrm{Q}}\right)- \\
& \mathcal{I}_{\left(M^{\prime}-M\right)(N+L)} \otimes \overline{\mathcal{E}}_{N+L}^{\otimes M}\left(\rho_{\mathrm{Q}}\right) \|_{1} .
\end{aligned}
$$

By further using the contractivity, we can upper bound from above the right part of the (B2) by:

$$
\begin{aligned}
& \| \tilde{\mathcal{I}}_{\left(M^{\prime}-M\right)(N+L)} \otimes \overline{\mathcal{F}}_{M(N+L)}\left(\rho_{\mathrm{Q}} \otimes \rho_{\mathrm{O}}\right)- \\
& \tilde{\mathcal{I}}_{\left(M^{\prime}-M\right)(N+L)} \otimes \overline{\mathcal{F}}_{N+L}^{\otimes M}\left(\rho_{\mathrm{Q}} \otimes \rho_{\mathrm{O}}\right) \|_{1},
\end{aligned}
$$

where $\tilde{\mathcal{I}}$ is the identity operator acting on the composite system QO, $\rho_{\mathrm{O}}=|0\rangle\langle 0|$ is the ground state of the oscillator and, as we have seen in section $\amalg$ IIA the quantum operations $\overline{\mathcal{F}}_{j(N+L)}$ are such that, for any integer $j$,

$$
\overline{\mathcal{E}}_{j(N+L)}\left(\rho_{\mathrm{Q}}\right)=\operatorname{Tr}_{\mathrm{O}}\left[\overline{\mathcal{F}}_{j(N+L)}\left(\rho_{\mathrm{Q}} \otimes \rho_{\mathrm{O}}\right)\right] .
$$

The memoryless map $\overline{\mathcal{F}}_{N+L}^{\otimes M}$ corresponds to apply $M$ times the map $\overline{\mathcal{F}}_{N+L}$ after the state of the oscillator has been reset to its ground state. Due to the triangle inequality we can bound from above the (B3) by:

$$
\begin{aligned}
& \| \tilde{\mathcal{I}}_{\left(M^{\prime}-M\right)(N+L)} \otimes \overline{\mathcal{F}}_{M(N+L)}\left(\rho_{\mathrm{Q}} \otimes \rho_{\mathrm{O}}\right)- \\
& \tilde{\mathcal{I}}_{\left(M^{\prime}-M\right)(N+L)} \otimes \overline{\mathcal{F}}_{N+L}^{\left(\pi_{0}\right)} \otimes \overline{\mathcal{F}}_{(M-1)(N+L)}\left(\rho_{\mathrm{Q}} \otimes \rho_{\mathrm{O}}\right) \|_{1}+ \\
& \| \tilde{\mathcal{I}}_{\left(M^{\prime}-M\right)(N+L)} \otimes \overline{\mathcal{F}}_{N+L}^{\left(\pi_{0}\right)} \otimes \overline{\mathcal{F}}_{(M-1)(N+L)}\left(\rho_{\mathrm{Q}} \otimes \rho_{\mathrm{O}}\right) \\
& \quad-\tilde{\mathcal{I}}_{\left(M^{\prime}-M\right)(N+L)} \otimes \overline{\mathcal{F}}_{N+L}^{\otimes M}\left(\rho_{\mathrm{Q}} \otimes \rho_{\mathrm{O}}\right) \|_{1} .
\end{aligned}
$$

where we set $\overline{\mathcal{F}}_{N+L}^{\left(\pi_{0}\right)} \equiv \overline{\mathcal{F}}_{N+L} \circ \pi_{0}$, being $\pi_{0}$ the quantum operation deleting the qubits-cavity correlations and resetting the cavity in its ground state (25). Let us consider the trace norm (B5). The first $(M-1)(N+L)$ uses of the two channels in (B5) are the same, bringing the system $\mathrm{QO}$ in the state $\rho_{\mathrm{QO}}$, so we can rewrite the (B5) as:

$$
\begin{aligned}
& \| \tilde{\mathcal{I}}_{\left(M^{\prime}-M\right)(N+L)} \otimes \overline{\mathcal{F}}_{N+L}\left(\rho_{\mathrm{QO}}^{\prime}\right)- \\
& \tilde{\mathcal{I}}_{\left(M^{\prime}-M\right)(N+L)} \otimes \overline{\mathcal{F}}_{N+L}\left[\pi_{0}\left(\rho_{\mathrm{QO}}^{\prime}\right)\right] \|_{1} .
\end{aligned}
$$

As we have seen in section IIIA for the expression in (24), it can be shown that the trace norm (B7) is smaller than:

$$
\| \rho_{Q \mathrm{Q}}^{\prime}-\rho_{\mathrm{Q}}^{\prime} \otimes|0\rangle\langle 0| \|_{1}<4 \sqrt{B} e^{-L \Gamma \tau / 2} .
$$

Now we turn to the trace norm (B6). The last not trivial $N+L$ uses for the two channels in the (B6) are described by the same quantum operation, so that by applying the contractivity of the trace norm with respect a quantum operation, we can upper bound the (B6) by:

$$
\begin{gathered}
\| \tilde{\mathcal{I}}_{\left(M^{\prime}-M+1\right)(N+L)} \otimes \overline{\mathcal{F}}_{(M-1)(N+L)}\left(\rho_{\mathrm{Q}} \otimes \rho_{\mathrm{O}}\right)- \\
\tilde{\mathcal{I}}_{\left(M^{\prime}-M+1\right)(N+L)} \otimes \overline{\mathcal{F}}_{N+L}^{\otimes(M-1)}\left(\rho_{\mathrm{Q}} \otimes \rho_{\mathrm{O}}\right) \|_{1}
\end{gathered}
$$

Note that this expression is formally equivalent to (B3), indeed it can be derived by the (B3) simply by replacing $M$ with $M-1$. By summarizing we have bounded the (B3) in the following manner:

$$
\begin{aligned}
& \| \tilde{\mathcal{I}}_{\left(M^{\prime}-M\right)(N+L)} \otimes \overline{\mathcal{F}}_{M(N+L)}\left(\rho_{\mathrm{Q}} \otimes \rho_{\mathrm{O}}\right)- \\
& \quad \tilde{\mathcal{I}}_{\left(M^{\prime}-M\right)(N+L)} \otimes \overline{\mathcal{F}}_{N+L}^{\otimes M}\left(\rho_{\mathrm{Q}} \otimes \rho_{\mathrm{O}}\right) \|_{1} \leq \\
& 4 \sqrt{B} e^{-L \Gamma \tau / 2}+ \\
& \| \tilde{\mathcal{I}}_{\left(M^{\prime}-M+1\right)(N+L)} \otimes \overline{\mathcal{F}}_{(M-1)(N+L)}\left(\rho_{\mathrm{Q}} \otimes \rho_{\mathrm{O}}\right)- \\
& \tilde{\mathcal{I}}_{\left(M^{\prime}-M+1\right)(N+L)} \otimes \overline{\mathcal{F}}_{N+L}^{\otimes(M-1)}\left(\rho_{\mathrm{Q}} \otimes \rho_{\mathrm{O}}\right) \|_{1}
\end{aligned}
$$

By recursively applying the (B10), up to $M-3$ times, we obtain:

$$
\begin{gathered}
\| \tilde{\mathcal{I}}_{\left(M^{\prime}-M\right)(N+L)} \otimes \overline{\mathcal{F}}_{M(N+L)}\left(\rho_{\mathrm{Q}} \otimes \rho_{\mathrm{O}}\right)- \\
\tilde{\mathcal{I}}_{\left(M^{\prime}-M\right)(N+L)} \otimes \overline{\mathcal{F}}_{N+L}^{\otimes M}\left(\rho_{\mathrm{Q}} \otimes \rho_{\mathrm{O}}\right) \|_{1} \leq \\
4 \sqrt{B}(M-2) e^{-L \Gamma \tau / 2}+ \\
\| \tilde{\mathcal{I}}_{\left(M^{\prime}-2\right)(N+L)} \otimes \overline{\mathcal{F}}_{2(N+L)}\left(\rho_{\mathrm{Q}} \otimes \rho_{\mathrm{O}}\right)- \\
\tilde{\mathcal{I}}_{\left(M^{\prime}-2\right)(N+L)} \otimes \overline{\mathcal{F}}_{N+L}^{\otimes(2)}\left(\rho_{\mathrm{Q}} \otimes \rho_{\mathrm{O}}\right) \|_{1}
\end{gathered}
$$


As for the (22), it can be shown that the last trace norm in (B11) is smaller than $4 \sqrt{B} e^{-L \Gamma \tau / 2}$, so that:

$$
\begin{aligned}
& \| \tilde{\mathcal{I}}_{\left(M^{\prime}-M\right)(N+L)} \otimes \overline{\mathcal{F}}_{M(N+L)}\left(\rho_{\mathrm{Q}} \otimes \rho_{\mathrm{O}}\right)- \\
& \quad \tilde{\mathcal{I}}_{\left(M^{\prime}-M\right)(N+L)} \otimes \overline{\mathcal{F}}_{N+L}^{\otimes M}\left(\rho_{\mathrm{Q}} \otimes \rho_{\mathrm{O}}\right) \|_{1} \leq \\
& 4 \sqrt{B}(M-1) e^{-L \Gamma \tau / 2}
\end{aligned}
$$

Because, as we say above, the left part of (B1) can be upper bounded by (B3), we can conclude that:

$$
\begin{aligned}
\left\|\overline{\mathcal{E}}_{M(N+L)}\left(\rho_{\mathrm{Q}}\right)-\overline{\mathcal{E}}_{N+L}^{\otimes M}\left(\rho_{\mathrm{Q}}\right)\right\|_{1} \leq \\
4(M-1) \sqrt{B} e^{-L \Gamma \tau / 2} .
\end{aligned}
$$

\section{Appendix C: Map for a single channel use}

We solve the Lindblad master equation (45), with the qubit prepared in the generic input state $\rho_{1}(0)$ of Eq. (38) and the oscillator initially in its ground state. During time evolution the overall system (qubit plus oscillator) resides in a three-dimensional subspace, spanned by the states $|0\rangle \equiv|g, 0\rangle,|1\rangle \equiv|g, 1\rangle,|2\rangle \equiv|e, 0\rangle$. We define $\rho_{i j} \equiv\left\langle i\left|\rho_{\mathrm{QO}}\right| j\right\rangle(i, j=0,1,2)$ and write the master equation in the $\{|0\rangle,|1\rangle,|2\rangle\}$ basis. We obtain the following differential equations:

$$
\begin{array}{r}
\dot{\rho}_{00}=\Gamma \rho_{11}, \\
\dot{\rho}_{01}=i \lambda \rho_{02}-\frac{\Gamma}{2} \rho_{01}, \\
\dot{\rho}_{02}=i \lambda \rho_{01}, \\
\dot{\rho}_{11}=i \lambda\left(\rho_{12}-\rho_{21}\right)-\Gamma \rho_{11}, \\
\dot{\rho}_{12}=i \lambda\left(\rho_{11}-\rho_{22}\right)-\frac{\Gamma}{2} \rho_{12}, \\
\dot{\rho}_{22}=-i \lambda\left(\rho_{12}-\rho_{21}\right),
\end{array}
$$

with initial conditions $\rho_{00}(t=0)=1-p, \rho_{01}(0)=0$, $\rho_{02}(0)=r, \rho_{11}(0)=0, \rho_{12}(0)=0, \rho_{22}(0)=p$. These linear equations can be solved in a standard way using the Laplace transform 30]. After tracing the solutions at time $\tau_{p}$ over the oscillator, we obtain the CPT map

$$
\mathcal{E}_{1}\left[\rho_{1}(0)\right]=\left[\begin{array}{cc}
1-p[h(\Gamma)]^{2} & r h(\Gamma) \\
r^{\star} h(\Gamma) & p[h(\Gamma)]^{2}
\end{array}\right],
$$

where

$$
h(\Gamma)=e^{-\Gamma \tau_{p} / 4}\left[\frac{\Gamma+z}{2 z} e^{z \tau_{p} / 4}-\frac{\Gamma-z}{2 z} e^{-z \tau_{p} / 4}\right],
$$

with $z=\sqrt{\Gamma^{2}-16 \lambda^{2}}$. This is an amplitude-damping channel as in Eq. (42), with parameter $\eta(\Gamma)=[h(\Gamma)]^{2}$.
[1] P. W. Shor, Phys. Rev. A 52, (1995) R2493.

[2] B. Schumacher and M. D. Westmoreland, Phys. Rev. A 56, (1997) 131.

[3] S. Lloyd, Phys. Rev. A 55, (1997) 1613.

[4] A. S. Holevo, IEEE Trans. Inf. Theory 44, (1998) 269.

[5] I. Devetak, IEEE Trans. Inf. Theory 51, (2005) 44.

[6] M. A. Nielsen and I. L. Chuang, Quantum computation and quantum information (Cambridge University Press, Cambridge 2000).

[7] G. Benenti, G. Casati, and G. Strini, Principles of quantum computation and information, vol. II (World Scientific, Singapore 2007).

[8] A. I. Lvovsky, B. C. Sanders, and W. Tittel, Nature Photonics 3 (2009) 706; Y. Kubo et al., Phys. Rev. Lett 105 (2010) 140502; T. Duty, Physics 3, (2010) 80.

[9] N. Gisin, Grgoire Ribordy, Wolfgang Tittel, and Hugo Zbinden Rev. Mod. Phys. 74, (2002) 145.

[10] C. H. Bennett and P. W. Shor, IEEE Trans. of Inf. Theory 44 (1998) 2724.

[11] C. Macchiavello and G. M. Palma, Phys. Rev. A 65, (2002) 050301(R).

[12] H. Hamada, J. Math. Phys. 43 (2002) 4382.

[13] G. Bowen and S. Mancini, Phys. Rev. A 69, (2004) 012306.

[14] V. Giovannetti, J. Phys. A 38, (2005) 10989.

[15] D. Kretschmann and R. F. Werner, Phys. Rev. A 72, (2005) 062323.

[16] N. Datta and T. C. Dorlas, J. Phys. A 40, (2007) 8147.

[17] M. B. Plenio and S. Virmani, Phys. Rev. Lett. 99, (2007)
120504; New J. Phys. 10, (2008) 043032.

[18] A. D'Arrigo, G. Benenti, and G. Falci, New J. Phys. 9, (2007) 310.

[19] D. Rossini et al. New J. Phys. 10 (2008) 115009.

[20] O. V. Pilyavets, V. G. Zborovskii, and S.Mancini, Phys. Rev. A 77, (2008) 052324.

[21] A. Bayat, D. Burgarth, S. Mancini, and S. Bose, Phys. Rev. A 77, (2008) 050306(R).

[22] G. Benenti, A. D'Arrigo, and G. Falci, Phys. Rev. Lett. 103, (2009) 020502.

[23] G. B. Lemos and G. Benenti, Phys. Rev. A 81, (2010) 062331.

[24] C. Lupo, V. Giovannetti and S. Mancini, Phys. Rev. Lett. 104, (2010) 030501.

[25] K. Banaszek, A. Dragan, W. Wasilewski, and C. Radzewicz, Phys. Rev. Lett. 92, (2004) 257901.

[26] Y. Makhlin, G. Schön, and A. Shnirman, Rev. Mod. Phys. 73, (2001) 357; E. Paladino, L. Faoro, G. Falci, and R. Fazio, Phys. Rev. Lett. 88, (2002) 228304; G. Falci, A. D'Arrigo, A. Mastellone, and E. Paladino, Phys. Rev. Lett. 94, (2005) 167002; G. Ithier et al., Phys. Rev. B 72, (2005) 134519.

[27] P. Meystre and M. Sargent III, Elements of quantum optics (4th Ed.) (Springer-Verlag, Berlin 2007).

[28] J.M. Raimond, M. Brune, and S. Haroche, Rev. Mod. Phys. 73, (2001) 565; S. Haroche and J.M. Raimond, Exploring the Quantum (Oxford University Press, Oxford 2006).

[29] A. Wallraff et al., Nature 431, (2004) 162; J. M. Fink 
et al., Nature 454, (2008) 315; S. M. Girvin et al., MH Devoret, and RJ Schoelkopf, Phys. Scr. T 137, (2009) 014012.

[30] X.-y. Chen, Quantum Inf. Process. 9, (2010) 451.

[31] H. Barnum, M. A. Nielsen, and B. Schumacher, Phys. Rev. A 57, (1998) 4153.

[32] B. W. Schumacher, Phys. Rev. A 54, (1996) 2614.

[33] B. W. Schumacher and M. A. Nielsen, Phys. Rev. A 54, (1996) 2629.

[34] I. Devetak and P. W. Shor, Comm. Math. Phys. 256, (2005) 287.

[35] G. Smith, Phys. Rev. A 78, (2008) 022306.

[36] One can also measure the reliability of entanglement transmission by means of the entanglement fidelity [32]. The capacity for entanglement transmission and the capacity for transmission of pure states in a subspace are equal [37] and given by the quantum capacity $Q$ defined in Eq. (2).

[37] H. Barnum, E. Knill, and M. A. Nielsen, IEEE Trans. Inf. Theory 46, (2000) 1317.

[38] P. Hausladen, R. Jozsa, B. Schumacher, M. Westmoreland, and W. K. Wootters, Phys. Rev. A 54, (1996) 1869.

[39] A. S. Holevo, Probl. Inf. Transm. 9, (1973) 177.

[40] Inequality (12) implies (11) since (see [6])

$$
\||\psi\rangle\langle\psi|-\sigma \|_{1} \geq 2(1-\sqrt{\langle\psi|\sigma| \psi\rangle}),
$$

where we have defined $\sigma=\overline{\mathcal{D}}^{(M)} \circ \overline{\mathcal{E}}_{N+L}^{\otimes M} \circ \overline{\mathcal{C}}^{(M)}(|\psi\rangle\langle\psi|)$.
[41] The quantity (13) is bounded from above by the sum of (the right side of) (12) and (16). By hypothesis the right side of (12) is less or equal than $\varepsilon / 2$ when $M>M_{0}$. The quantity (16) is bounded by $h(M-1) c^{-L}$; because of $\lim _{M \rightarrow \infty} h(M-1) c^{-\varepsilon M}=0$, it there exists $\tilde{M}$ such that $\forall M>\tilde{M} \Rightarrow h(M-1) c^{-\varepsilon M} \leq \varepsilon / 2$. Then $M_{0}^{\prime}=$ $\max \left\{M_{0}, \tilde{M}\right\}$.

[42] Focus on Quantum Dissipation in Unconventional Environments, M. Grifoni and E. Paladino Eds., New J. Phys. 10 (2008); F. Cavaliere et al., New J. Phys. 10, (2008) 115004; A. Garg et al., Jour. Chem. Phys. 83, (1985) 4491; F. Plastina and G. Falci, Phys. Rev. B 67, (2003) 224514.

[43] V. Giovannetti and R. Fazio, Phys. Rev. A 71, (2005) 032314.

[44] G. Lindblad, Commun. Math. Phys. 48, (1976) 119; V. Gorini, A. Kossakowski, and E.C.G. Sudarshan, J. Math. Phys. 17, (1976) 821.

[45] P. Facchi and S. Pascazio, J. Phys. A: Math. Theor. 41, (2008) 493001.

[46] To diagonalize the systems density matrix in order to calculate their von Neumann entropies, we used "Routines for the diagonalization of complex matrices", T. Hahn, arXiv:physics/0607103 2 .

[47] B. Groisman, S. Popescu, and A. Winter, Phys. Rev. A 72, (2005) 032317. 